\title{
Length scale and manufacturability in density-based topology optimization
}

\author{
Lazarov, Boyan Stefanov; Wang, Fengwen; Sigmund, Ole
}

Published in:

Archive of Applied Mechanics

Link to article, DOI:

10.1007/s00419-015-1106-4

Publication date:

2016

Document Version

Peer reviewed version

Link back to DTU Orbit

Citation (APA):

Lazarov, B. S., Wang, F., \& Sigmund, O. (2016). Length scale and manufacturability in density-based topology optimization. Archive of Applied Mechanics, 86(1-2), 189-218. https://doi.org/10.1007/s00419-015-1106-4

\section{General rights}

Copyright and moral rights for the publications made accessible in the public portal are retained by the authors and/or other copyright owners and it is a condition of accessing publications that users recognise and abide by the legal requirements associated with these rights.

- Users may download and print one copy of any publication from the public portal for the purpose of private study or research.

- You may not further distribute the material or use it for any profit-making activity or commercial gain

- You may freely distribute the URL identifying the publication in the public portal

If you believe that this document breaches copyright please contact us providing details, and we will remove access to the work immediately and investigate your claim. 


\title{
Length scale and manufacturability in density based topology optimization
}

\author{
Boyan S. Lazarov • Fengwen Wang · Ole \\ Sigmund
}

Received: date / Accepted: date

\begin{abstract}
Since its original introduction in structural design, density based topology optimization has been applied to a number of other fields like microelectromechanical systems, photonics, acoustics and fluid mechanics. The methodology has been well accepted in industrial design processes where it can provide competitive designs in terms of cost, materials and functionality under a wide set of constraints. However, the optimized topologies are often considered as conceptual due to loosely defined topologies and the need of post processing. Subsequent amendments can affect the optimized design performance and in many cases can completely destroy the optimality of the solution. Therefore, the goal of this paper is to review recent advancements in obtaining manufacturable topology optimized designs. The focus is on methods for imposing minimum and maximum length scale, and ensuring manufacturable, well defined designs with robust performances. The overview discusses the limitations, the advantages and the associated computational costs. The review is completed with optimized designs for minimum compliance, mechanism design and heat transfer.
\end{abstract}

Keywords Topology optimization · Length scale $\cdot$ Manufacturability $\cdot$ Regularization

\section{Introduction to topology optimization}

The topology optimization process aims at distributing a specified amount of material in a given design domain by minimizing an objective function and fulfilling a

\author{
B. S. Lazarov \\ Technical University of Denmark \\ Nils Koppels Allé, Building 404 \\ 2800 Kgs. Lyngby, Denmark \\ E-mail: bsl@mek.dtu.dk \\ F. Wang \\ E-mail: fwan@mek.dtu.dk \\ O. Sigmund \\ E-mail: sigmund@mek.dtu.dk
}


set of constraints. Here the focus is on density based formulations which in general can be written as

$$
\begin{aligned}
\min _{\rho} & : J(\rho, u) \\
\text { s.t. } & : r(\rho, u)=0, \quad u \in \mathcal{U}_{\mathrm{ad}} \\
& g_{i}(\rho, u) \leq 0, \quad i=1 \ldots N_{g} \\
& \rho \in \mathcal{D}_{\mathrm{ad}}
\end{aligned}
$$

where $J(\rho, u)$ is an objective function (weight, material volume, mechanical compliance, displacements or velocities at a subdomain of the design domain), $r(\rho, u)=$ 0 represents the residual of the equations modelling the physical system with solution $u \in \mathcal{U}_{\mathrm{ad}}$, and $\left\{g_{i}(\cdot) \leq 0, i=1 \ldots N_{g}\right\}$ is a set of constraints. The design domain is an open bounded domain in $\mathbb{R}^{d}$ with Lipschitz boundary $\Gamma=\partial \Omega$. The material density field in represented by a bounded function $\rho \in L^{\infty}(\Omega)$ and $\rho_{\min } \leq$ $\rho(\mathbf{x}) \leq \rho_{\max }, \mathbf{x} \in \Omega$, i.e., $\mathcal{D}_{\text {ad }}=\left\{\rho \in L^{\infty}(\Omega): \rho_{\min } \leq \rho(\mathbf{x}) \leq \rho_{\max }, \mathbf{x} \in \Omega\right\}$. Usually the upper bound is set to one and the lower bound to zero.

If the material density field is equal to one at a point in the design domain, the point is occupied with material. The material density field is equal to zero at the void regions. The discrete $0 / 1$ problem is relaxed in order to utilize gradient based optimization techniques and the material density is allowed to take intermediate values. Such a relaxation requires some interpretation of the gray transition regions. Removing them in a post processing step might affect the optimality of the solution and might violate the design constraints. Hence, reviewing the techniques for avoiding the post processing step while obtaining well performing and manufacturable designs close to $0 / 1$ is the main goal of this article. The focus is on the so-called three field density representation where the density field representing the design is obtained by a series of transformations in order to restrict the design space and ensure manufacturability of the design.

\subsection{Interpolation schemes in topology optimization}

The most popular problem in topology optimization is minimum compliance design originally formulated by Bendsøe and Kikuchi in [18. The idea is to distribute material in a design domain in order to minimize the compliance of a mechanical system and the material properties are obtained using a homogenization approach. Later, the so-called SIMP (Simplified Isotropic Material with Penalization) 17. approach has been introduced in order to reduce the complexity of the original homogenization formulation. The SIMP formulation has been utilized in many following papers to improve convergence to 0/1 solution, e.g., 167] and [107, and physical justification is presented later in [19. For linear elasticity, SIMP relates the modulus of elasticity $E(\mathbf{x}), \mathbf{x} \in \Omega$ and the material density field by the power law given as

$$
E(\mathbf{x})=E_{\min }+\rho(\mathbf{x})^{p}\left(E_{0}-E_{\min }\right)
$$

where, $E_{\min }$ is taken to be a small value larger than zero in order to ensure existence of the solution of the linear elastic problem, $E_{0}$ is the modulus of elasticity for the solid material, and $p$ is a penalization parameter. For $p=1$ the minimum compliance problem is convex and possesses a unique solution with large gray 
regions. Increasing the penalty parameter improves the contrast, and a value of $p=3$ usually ensures good convergence to $0 / 1$ designs. It should be pointed out that the penalization works only for problems with constraints which directly or indirectly limit the material volume.

The SIMP interpolation scheme results in zero gradients for all regions with zero density $\rho=0$. An alternative scheme, the so-called RAMP (Rational Approximation of Material Properties) interpolation is proposed in the literature [130, and can be utilized for problems where SIMP is undesirable. For general discussion and comparison the readers are referred to [131] and the recent review papers on topology optimization [126] and 37. Designs close to $0 / 1$ can be obtained by tuning the parameters for the selected material optimization scheme. Recently, an interpolation scheme for nonlinear materials was proposed in 148, where the interpolation is performed between two material potentials rather than between two material properties. Such an interpolation avoids the numerical instabilities observed for optimization problems with large deformations.

Another alternative to the implicit penalization provided by SIMP or RAMP is to utilize explicit penalization of the intermediate values by adding the following term to the objective function, e.g., [5] and [9].

$$
\alpha \int_{\Omega} \rho(1-\rho) \mathrm{d} \Omega
$$

Explicit penalization is often utilized for wave propagation optimization problems 144] where the traditional interpolation schemes cannot penalize the intermediate values, e.g., 86. The main difficulty consists in determining the penalization parameter $\alpha$. The explicit penalization can be introduced to the optimization problem as a constraint. However, in this case the difficulty for selecting $\alpha$ is transferred to the selection of the constraint value. A variation of the explicit penalization is proposed in [22] where the density $\rho$ in Equation 6 is replaced with a density average which imposes a length scale on the design, however, with the same challenges in selecting $\alpha$ as for the expression given by Equation 6

\subsection{Discretization schemes}

The optimization is performed iteratively using the so called nested formulation, where at each optimization step the state problem is solved, the objective and the constraints are computed from the obtained state solution, the gradients of the objective and the constraints with respect to the parametrization of the design field are computed using adjoint analysis, and the design field is updated based on the gradients. The areas of applicability include problems in linear elasticity, heat transfer, fluid mechanics, vibrations, acoustics, electromagnetics, nanomechanics. The state problem can be discretized and solved using almost any of the available numerical methods for solving partial differential equations like Finite Element Method (FEM), Finite Volume (FV), Finite Difference (FD), Discontinuous Galerkin (DG-FEM), etc.

The material density field usually follows the discretization of the state solvers. For a FEM discretization the density field is mostly represented using constant values within each finite element. For a FD discretization a constant value is associated with the cell surrounding each node. For FV and DG-FEM a constant 
value is often utilized for each control volume. The density field discretization can be decoupled from the discretization of the state problem 66] which might result in reduction of the optimization time.

\subsection{Regularization in topology optimization}

Minimizing the compliance of a structure using material density associated with each finite element results in two major issues: 1) mesh dependency and 2) checkerboard patterns. Mesh dependency can be observed by refining the design domain for a design obtained with a coarse mesh and running the optimization on the refined mesh. The optimization might converge to a completely different and more complex topology than the one obtained using the coarse mesh. Such problems are referred to as ill-posed in the mathematical literature since they do not posses a solution. Checkerboard patterns appear due to bad numerical properties of the discretization and they do not represent optimal solutions [38, [77.

Existence of the solution is provided by regularizing the optimization problem, i.e., by restricting the solution space. A number of regularization schemes have been proposed in the literature: perimeter control [70], sensitivity filtering [123, and mesh independent density filtering [23, [27]. Here the focus is on density filtering combined with subsequent transformations. For discussion of the other regularization techniques the reader is referred to [126].

\subsection{Alternative density based approaches}

A number of alternative density based approaches have been proposed in the research literature. The most popular of them is the level set method which utilize a level set function to represent the shape of the design [7, [8] and [151]. Recent overview of different level-set methods can be found in [39] and comparison to density based approaches in 126. As outlined several times in 126, the projection based three field formulation which is in focus in this article becomes more and more similar to the level set methods. Hence, many techniques developed for one of the approaches can often be easily transferred to the other one. Other alternatives include the topological derivative method [127, the phase field method 24, evolutionary approaches [156], etc.

\section{Density filters}

Density filtering in topology optimization can be written in the form of a convolution integral [23] given as

$$
\widetilde{\rho}(\mathbf{x})=\int_{B} w(\mathbf{x}-\mathbf{y}) \rho(\mathbf{y}) \mathrm{d} \mathbf{y}
$$

where $\widetilde{\rho}$ is the filtered density field, $\rho$ is the original design field and $w(\cdot)$ is a weighting function. The different density filters differ mainly in their weighting 
functions. The weighting function for the classical density filter [23] is the Hat function given by

$$
w(\mathbf{x})= \begin{cases}\frac{1}{w_{w}}\left(1-\frac{|\mathbf{x}|}{R_{f}}\right), & |\mathbf{x}| \leq R_{f} \\ 0, & |\mathbf{x}|>R_{f}\end{cases}
$$

where the normalization factor $w_{w}$ is obtained from the condition $\int w(\mathbf{x}) \mathrm{d} \mathbf{x}=1$. The support domain of the filter function $B=\operatorname{supp}(w)$ is circular in $2 \mathrm{D}$ and spherical in $3 \mathrm{D}$ with radius $R_{f}$. The integral condition for determining the normalization factor ensures that the filter preserves as the total volume.

In general the support domain can take any shape which will determine the properties of the projections discussed in the following section. For density filters with finite support domain the design domain is extended by dilating it with the filter support, i.e., $\Omega^{*}=\{\mathbf{x}: \mathbf{y} \in \Omega \wedge w(\mathbf{x}-\mathbf{y}) \neq 0\}$, where $\Omega^{*}$ is the extended design domain. The values of the design field outside the design domain $\rho(\mathbf{x}), \mathbf{x} \in \Omega^{*} \backslash \Omega$ can be either prescribed in order to ensure a particular behavior of the filtered field around the boundaries of $\Omega$, or they can be included in the optimization problem. Often the design domain is not extended and in this case the filter behavior around $\partial \Omega$ differs from the filter behavior in the interior of the design domain.

In 27] an exponential filter function is proposed which does not posses finite support. In this case the function support can be truncated. Alternatively some assumptions about the input design field outside the design domain have to be provided in advance. The convolution integral Equation 8 is extended over $\mathbb{R}^{d}$, where $d=2,3$ is the dimensionality of the problem.

An alternative to the convolution integral formulation, based on the solution of a partial differential equation (PDE), has been proposed first for sensitivity filtering in [87 and later extended to density filtering in 93] and 79. The idea is to obtain the filtered field as the solution of a PDE given as

$$
-\nabla^{\mathrm{T}} r_{f}(\mathbf{x})^{2} \nabla \widetilde{\rho}(\mathbf{x})+\widetilde{\rho}(\mathbf{x})=\rho(\mathbf{x}), \quad \mathbf{x} \in \Omega
$$

The parameter $r_{f}(\mathbf{x})$ controls the effective width of the filter. It can be fixed or allowed to vary along the design domain. Larger $r_{f}$ results in larger gray transition regions and smaller $r_{f}$ results in sharper transition between void and solid. The filter is volume preserving for Neumann boundary conditions, $\partial \widetilde{\rho} / \partial \mathbf{n}=0$ on $\partial \Omega$, where $\mathbf{n}$ is the outward normal to the design domain boundary. The main advantages compared to classical filters are the lower computational cost [2], the re-utilization of solvers developed for the state problems 93, relatively easy parallelization for large scale problems [1, utilization of different filter parameters in different parts of the design domain, and the possibility of controlling the behavior of the filtered field without extending the design domain. The Neumann boundary conditions can be replaced with Dirichlet boundary conditions which prescribe exact boundary values for the filtered field 90. The solution of the PDE can be expressed as the convolution of the input design field $\rho(\mathbf{x})$ and the Green's functions [159] of the boundary value problem given by Equation 9 The filter support domain coincides with the design domain. The computational cost of the PDE filter using multigrid solvers is $\mathcal{O}(n)$ [142.

A relation between the length parameter $r_{f}$ in Equation 9 and the filter radius $R_{f}$ in Equation 8 can be obtained by matching the second central moments of the Hat function and the Green's function for the PDE filter on unbounded 
domain [93. The condition leads to similar filter fields and gray levels. The length parameter $r_{f}$ can be related to $R_{f}$ as follows

$$
r_{f}=\frac{R_{f}}{2 \sqrt{3}}
$$

Following the idea of state solver re-utilization in the filtering process, the implementation of topology optimization problems with transient behavior, e.g., 110 would benefit from transient PDE filtering schemes. Based on regularization strategies utilized in image processing [16] a time dependent non-linear diffusion regularization scheme in topology optimization is proposed in [150] where the focus is mainly on reducing the gray transition regions by tuning the diffusion coefficient along the design edges. In [78, a time dependent diffusion is utilized for regularizing a level-set like topology optimization formulation. State solver reutilization and the parallelization benefits for density based topology optimization of time dependent problems in photonics are demonstrated in [45. The filtered field is obtained as the solution $\widetilde{\rho}(\mathbf{x}, t)$ of the following diffusion equation

$$
\frac{\partial \widetilde{\rho}}{\partial t}=\triangle \widetilde{\rho}
$$

with prescribed boundary conditions and initial condition $\widetilde{\rho}(t=0, \mathbf{x})=\rho(\mathbf{x}), \mathbf{x} \in$ $\Omega$. The solution can be represented in the form of Equation 7 where the filter function is a Gaussian kernel [16

$$
w_{\sigma}(\mathbf{x})=\frac{1}{2 \pi \sigma^{2}} \exp \left(-\frac{|\mathbf{x}|^{2}}{2 \sigma^{2}}\right)
$$

with filter parameter $\sigma$ which scales with time as $\sigma=\sqrt{2 t}$.

The time dependent heat equation as well as the PDE filter discussed above diffuse the design field in an isotropic manner. Anisotropic filters which favor a particular direction and stops information propagation in others can be obtained by replacing the scalar coefficients with tensor quantities. Anisotropic filtering can be achieved in the classical filter given by Equation 7 by selecting weighting function with support different than circular.

In all cases the filter acts as a low pass filter to the original design field $\rho$. For regular rectangular domains low pass filtering can effectively be constructed by Fourier transform, e.g., 128. The idea is applied in density based topology optimization in [88. The design field $\rho(\mathbf{x})$ is transformed in the frequency domain using 1D, 2D or 3D discrete Fourier transform, and then convoluted with a filter function. The filter function in this case is defined entirely in the frequency domain. The filter is given by the following three steps

$$
\begin{array}{r}
\rho(\boldsymbol{\omega})=\mathcal{F}[\rho(\mathbf{x})] \\
\widetilde{\rho}(\boldsymbol{\omega})=w(\boldsymbol{\omega}) \rho(\boldsymbol{\omega}) \\
\widetilde{\rho}(\mathbf{x})=\mathcal{F}_{i}[\widetilde{\rho}(\boldsymbol{\omega})]
\end{array}
$$

where $\mathcal{F}$ denotes the Fourier transform and $\mathcal{F}_{i}$ its inverse. Practical realizations of the above filter benefit significantly from the tuned fast numerical implementations based on the Fast Fourier Transform (FFT). The appearance of artifacts around the boundaries of the design domain can be decreased by padding (extending) 
the domain with zeros. Extending the domain with patterns different than zero can control design field values around the borders of the design domain. Any post processing of the filtered design, e.g., finding derivatives of any order, can be easily performed in the frequency domain [140. The larger freedom in constructing the filter weighting function in the frequency domain can be utilized for controlling the maximum length scale as well as the spacing between the solid and the void regions [88. These aspects are discussed in subsection 8.2

The main limitation in utilizing FFT filters in topology optimization is the requirement for rectangular shape of the design domain. Irregular domains can be padded with zeros or prescribed patterns. Alternatively an effective algorithm for calculating the convolution integral given by Equation 7 can be based on the fast multipole method (FMM) [57. Such a possibility remains to be investigated.

\section{Projections}

Gray regions always exist in the solution due to the relaxation of the original $0 / 1$ problem. Penalization schemes can decrease their appearance to some extend, however, some post processing of the design might be necessary if the final goal of the optimization process it pure black and white design. Aiming at reducing the intermediate transition regions between solid and void, various alternative filtering schemes based on projections [61], on morphological operators [124] and more recently on Pythagorean means [135] have been proposed in the literature.

The projection scheme proposed in [61] can impose length scale either on the solid phase or on the void phase 124 . The idea is to filter the design field using filter with finite support and project all values of $\widetilde{\rho}$ above zero to one. Such a projection step ensures that the smallest solid feature in the design will be equal or larger than the filter support, i.e., the solid design can be painted with a circle in $2 \mathrm{D}$ or a sphere in $3 \mathrm{D}$ with radius $R_{f}$. The projection step is given as

$$
\widehat{\widetilde{\rho}}=\mathrm{H}(\widetilde{\rho})
$$

where $H(\cdot)$ is the Heaviside function. The Heaviside function is not differentiable, therefore, for gradient based optimization it is replaced with a parameter dependent expression

$$
\mathrm{H}(\widetilde{\rho}) \approx 1-\exp (-\beta \widetilde{\rho})+\widetilde{\rho} \exp (-\beta)
$$

The above expression approaches the Heaviside function for $\beta \rightarrow \infty$. Length scale on the void phase can be obtained by applying the Heaviside projection to $1-\widetilde{\rho}$, i.e., the projected field is obtained as

$$
\widehat{\widetilde{\rho}}=1-\mathrm{H}(1-\widetilde{\rho})
$$

In 124 so-called morphological operators [109] are applied as filters in topology optimization. The two basic morphological operators, erode and dilate, are defined originally for black and white images. The erode operator sets a point to zero if any point in the neighborhood covered by the filter support has zero value and one otherwise. The dilate operator sets a point to one if any point in the neighborhood covered by the filter support has value one and zero otherwise, i.e., the dilate operator deposits material along the perimeter of the design. For grayscale images the erode operator sets the value of a point to the maximal value in 
the neighborhood covered by the filter support and the dilate operator sets it to the minimum. The dilate operator is given as

$$
\widehat{\widetilde{\rho}}=\frac{1}{\beta} \log \left(\int_{B} w(\mathbf{x}-\mathbf{y}) \mathrm{e}^{\beta \rho(\mathbf{y})} \mathrm{d} \mathbf{y}\right)
$$

and the erode operator is defined as

$$
\widehat{\widetilde{\rho}}=1-\frac{1}{\beta} \log \left(\int_{B} w(\mathbf{x}-\mathbf{y}) \mathrm{e}^{\beta(1-\rho(\mathbf{y}))} \mathrm{d} \mathbf{y}\right)
$$

Both operators utilize Kreisselmeier-Steinhauser [84 approximation to the min / max operator.

Recently an alternative definition to erode/dilate filters based on Pythagorean means have been proposed in [135. The erode/dilate filters based on harmonic mean can be written in integral form as

$$
\begin{array}{r}
\frac{1}{\widehat{\widetilde{\rho}}+\beta}=\int_{B} \frac{w(\mathbf{x}-\mathbf{y})}{\rho(\mathbf{y})+\beta} \mathrm{d} \mathbf{y} \\
\frac{1}{1-\widehat{\widetilde{\rho}}+\beta}=\int_{B} \frac{w(\mathbf{x}-\mathbf{y})}{1-\rho(\mathbf{y})+\beta} \mathrm{d} \mathbf{y}
\end{array}
$$

where the parameter $\beta$ controls the sharpness of the projection.

The above projections rely on the finite filter support to impose length scale on the optimized designs. The original Heaviside projection 61 projects the transition region to zero and one, however, due to the continuity of the projected field $\widehat{\widetilde{\rho}}$ the transition from void to solid passes always through intermediate values. In [135] the Hat filter is replaced with uniform weights (constant value) inside the filter support. This provides sharper transition due to the allowance of discontinuities in the final design $\widehat{\widetilde{\rho}}$. For well penalized optimization problems a constant filter results in sharp black and white designs, however, for problems where the objective benefits from the appearance of intermediate design values the above projection techniques will result in gray in the final design. The projections do not penalize the designs. They only allow for sharper transition. These effects can be clearly observed on designs shown in Table 3 and Table 4 Another important observation for the morphological filters is that they project to the $\mathrm{min} / \mathrm{max}$ value within the filter support which is different than zero or one in the relaxed optimization problem.

Any of the above projection techniques for PDE/FFT/FMM filters, with support coinciding with the design domain, will result in design domain occupied entirely by one of the phases. Such behavior can be alleviated by thresholding the design or the filtered field with threshold between zero and one [158. It should be pointed out that in this case the length scale (mesh independence) imposed on the design is lost. These effects are discussed in details in 147 where the expression for the intermediate threshold Heaviside projection, which is utilized here, is proposed in the form

$$
\mathrm{H}_{\eta}(\widetilde{\rho})=\frac{\tanh (\beta \eta)+\tanh (\beta(\widetilde{\rho}-\eta))}{\tanh (\beta \eta)+\tanh (\beta(1-\eta))}
$$

with parameter $\beta$ playing the same role as in Equation 16 and $\eta$ denoting the threshold level. 


\section{Three field density representation}

As discussed in the previous sections the final density field $\widehat{\widetilde{\rho}}$ is obtained by series of transformations applied on the original design field $\rho$. The basic transformations are filtering and projection and the chain of transformations can be written as $\rho \rightsquigarrow \widetilde{\rho} \rightsquigarrow \widehat{\widetilde{\rho}}$ which will be referred to as the three field density representation. The concept provides a powerful tool for controlling the final topology and acts as a basic building block for more complex control strategies summarized in Table 1

In 62 length scales on both phases are obtained by the utilization of two design fields. The first one $\bar{\rho}_{1}$ represents the void and the second one $\bar{\rho}_{2}$ represents the solid. They are obtained by using the three field representation from two design fields $\rho_{1}$ and $\rho_{2}$, respectively. The final design field is obtained as average of the two fields which produces gray transition regions. Various extensions are presented in 64, 69, and 63 where combinations of multiple phases and Heaviside projection with fixed support shape are utilized to control particle topologies, positions and distances between them in the design of structures and materials. In [163] the theoretical development is demonstrated in the design of 3D woven lattices.

In 121] the void phase is represented as a union of discrete shape projections. The idea is extended in [146] for the design of photonic crystal wave guides and recently it is applied in [11] to density based topology optimized designs consisting of a union of elements with prescribed pattern. In general several strategies are applicable to density based formulations:1) the design can be obtained by a union of prescribed number of discrete shapes; 2) the original design field can be represented as a union of discrete shapes and the final one is obtained using the three field representation; 3) the original design field is filtered or projected using filters with compact support defined by the desired shape. The first case might result in a non-differentiable formulation, e.g., [160. The second provides limits on the optimization space as each shape is defined by a few parameters and the total number of shapes is fixed. This can result in faster convergence, however, the design might be poorly performing compared to designs obtained with larger number of parameters. The third approach keeps the complete freedom of the topology optimization and the only limit is imposed by the discretization process.

Another recent extension 34] of the three field representation utilizes the gradient of the projected density field $\bar{\rho}$ for optimizing structures with a coating layer. The boundary of the solid phase can be identified by using the gradients of the density. As the transition from solid to void can become very sharp and is mesh dependent, the density field is filtered first and then differentiated. Inside solid or void regions the norm of the gradient vector field $|\nabla \tilde{\bar{\rho}}|$ is zero or close to zero. Close to the transition regions the norm is different than zero, which is utilized for defining a coating layer around the solid region. Then Heaviside projection of $|\nabla \widetilde{\bar{\rho}}|$ combined with control on the magnitude of the gradient norm provides density interpolation for the coating material. A similar extension is utilized in the design of piezo modal transducers with prescribed electrode gaps in [41].

As discussed in detail in [147] and demonstrated in Table 4 a density field based on Heaviside projection combined with filtering with finite support provides length scale either on the void or the solid phase, i.e., designs with length scale imposed on the solid can posses extremely small features in the void regions, and vice verse. These small features might be mesh dependent and observable by refin- 
Table 1 Overview of the different filter-projection schemes for density based topology optimization

\begin{tabular}{|c|c|c|c|c|}
\hline Name & Variable & Operations & $\begin{array}{l}\text { Regularization and length } \\
\text { scale }\end{array}$ & References \\
\hline SIMP & $\rho$ & $\rho$ & sensitivity filter & 123 \\
\hline SIMP & $\widetilde{\rho}$ & $\rho \rightsquigarrow \widetilde{\rho}$ & density filter & 23,27 \\
\hline Projection & $\widehat{\widetilde{\rho}}$ & $\rho \rightsquigarrow \widetilde{\rho} \rightsquigarrow \widehat{\widetilde{\rho}}$ & length scale on one phase & 61 \\
\hline $\begin{array}{l}\text { Multiple phase } \\
\text { projection }\end{array}$ & $\bar{\rho}$ & $\left(\begin{array}{c}\rho_{1} \\
\rho_{2}\end{array}\right) \rightsquigarrow\left(\begin{array}{c}\widetilde{\rho}_{1} \\
\widetilde{\rho}_{2}\end{array}\right) \rightsquigarrow\left(\begin{array}{c}\widetilde{\widetilde{\rho}}_{1} \\
\widetilde{\widetilde{\rho}}_{2}\end{array}\right) \rightsquigarrow \bar{\rho}$ & $\begin{array}{l}\text { length scale on both phases } \\
\text { with gray transition }\end{array}$ & 62 \\
\hline $\begin{array}{l}\min / \max \text { multi- } \\
\text { ple projections }\end{array}$ & $\widehat{\widetilde{\rho}}_{i}, i=e, i, d$ & $\rho \rightsquigarrow \widetilde{\rho} \rightsquigarrow\left\{\widehat{\widetilde{\rho}}_{e}, \widehat{\widetilde{\rho}}_{i}, \widehat{\widetilde{\rho}}_{d}\right\}$ & $\begin{array}{l}\text { length scale on the blueprint } \\
\text { only if the topology be- } \\
\text { tween the realizations does } \\
\text { not change }\end{array}$ & 147 \\
\hline $\begin{array}{l}\text { Robust objective } \\
\text { with random uni- } \\
\text { form projections }\end{array}$ & $\widehat{\widetilde{\rho}}_{i}, i=1, \ldots, \infty$ & $\rho \rightsquigarrow \widetilde{\rho} \rightsquigarrow\left(\widehat{\widetilde{\rho}}_{i}, i=1, \ldots, \infty\right)$ & same as $\min / \max$ & 90 \\
\hline $\begin{array}{l}\text { Robust } \quad \text { objective } \\
\text { with } \quad \text { random } \\
\text { spatially } \\
\begin{array}{l}\text { projections }\end{array}\end{array}$ & $\widehat{\widetilde{\rho}}_{i}, i=1, \ldots, \infty$ & $\rho \rightsquigarrow \widetilde{\rho} \rightsquigarrow\left(\widetilde{\widetilde{\rho}}_{i}, i=1, \ldots, \infty\right)$ & same as $\min / \max$ & 122 \\
\hline Double projection & $\overline{\bar{\rho}}_{i}, i=1, \ldots, \infty$ & $\begin{aligned} \rho & \rightsquigarrow \widetilde{\rho} \rightsquigarrow \widehat{\widetilde{\rho}} \rightsquigarrow \\
& \rightsquigarrow \widetilde{\bar{\rho}} \rightsquigarrow\left\{\overline{\bar{\rho}}_{i}, i=1, \ldots, \infty\right\}\end{aligned}$ & same as $\min / \max$ & 32 \\
\hline $\begin{array}{l}\text { Discrete } \quad \text { Object } \\
\text { Projection }\end{array}$ & $\bar{\rho}$ & $\rho \rightsquigarrow\left(\begin{array}{c}\widetilde{\rho}_{L} \\
\widetilde{\rho}_{E}\end{array}\right) \rightsquigarrow\left(\begin{array}{c}\widetilde{\widetilde{\rho}}_{L} \\
\widetilde{\widetilde{\rho}}_{E}\end{array}\right) \rightsquigarrow \bar{\rho}$ & $\begin{array}{l}\text { length scale between the in- } \\
\text { clusions and on the inclusion }\end{array}$ & 69], 63 \\
\hline $\begin{array}{l}\text { Minimum length } \\
\text { scale }\end{array}$ & $\bar{\rho}$ & $\rho \rightsquigarrow\{\widetilde{\rho}, \nabla \widetilde{\rho}\} \rightsquigarrow \widehat{\widetilde{\rho}}$ & $\begin{array}{l}\text { black and white design with } \\
\text { length scale on both phases }\end{array}$ & 166 \\
\hline Coating projection & $\bar{\rho}, \overline{\nabla \widetilde{\rho}}$ & $\rho \rightsquigarrow \widetilde{\rho} \rightsquigarrow \widetilde{\widetilde{\rho}} \rightsquigarrow \widetilde{\bar{\rho}} \rightsquigarrow \overline{\nabla \widetilde{\bar{\rho}}}$ & length scale on the coating & 34 \\
\hline
\end{tabular}


ing the mesh. This often results in lack of manufacturability, extremely sensitive performance due to manufacturing and exploitation uncertainties, stress concentration, etc. The projection does not provide penalization to the optimization problem and it cannot avoid the appearance of intermediate densities. This effect is pronounced especially well in the design of heat sink Table 3 Hence, in general topology optimized designs based solely on Heaviside projection requires post processing which might destroy completely the effect of the optimization. More complex extensions, which avoid the post processing are discussed in section 7 and section 8 They are built on the three field formulation or additional constraints and provide length scale on both phases 166 and robust designs performance 147, 90, and [122].

\section{Numerical examples}

In order to demonstrate the effect of the different projection and filtering strategies a set of selected topology optimization problems are shown in Figure 1 and described below. The set consists of: 1) L-bracket optimization 94, 2) compliant mechanism design [123, and 3) heat sink optimization problem [20]. The same or similar examples have been considered in [147] and subsequent papers. The design domains are discretized using first order finite elements [171. The design field $\rho$, the filtered field $\widetilde{\rho}$ and the projected field $\bar{\rho}$ are discretized using constant values within each finite element. The collected values are stored in vectors denoted as $\boldsymbol{\rho}, \widetilde{\rho}$ and $\overline{\boldsymbol{\rho}}$, respectively.

The L-bracket optimization problem can be written in discrete form as

$$
\begin{array}{cl}
\min _{\rho}: J=\mathbf{f}^{\top} \mathbf{u} \\
\text { s.t. }: \mathbf{K}(\boldsymbol{\rho}) \mathbf{u}=\mathbf{f} \\
\\
\overline{\boldsymbol{\rho}}^{\top} \mathbf{v} \leq V^{*} \\
\mathbf{0} \leq \boldsymbol{\rho} \leq \mathbf{1}
\end{array}
$$

where $\mathbf{K}$ is the stiffness matrix obtained using finite element discretization, $\mathbf{f}$ is a vector consisting of the input supplied to the system, $V^{*}$ denotes the material volume, and the vector $\mathbf{v}$ consists of the volumes of all elements. The gradients with respect to the projected densities are obtained using adjoint analysis and are given as

$$
\frac{\partial J}{\partial \overline{\boldsymbol{\rho}}_{j}}=-\mathbf{u}^{\top} \frac{\partial \mathbf{K}}{\partial \overline{\boldsymbol{\rho}}_{j}} \mathbf{u}
$$

The gradients with respect to the original design field are obtained using the chain rule

$$
\frac{\partial J}{\partial \boldsymbol{\rho}}=\frac{\partial J}{\partial \bar{\rho}} \frac{\partial \overline{\boldsymbol{\rho}}}{\partial \widetilde{\boldsymbol{\rho}}} \frac{\partial \widetilde{\boldsymbol{\rho}}}{\partial \boldsymbol{\rho}}
$$

The compliant mechanism optimization problem is defined as

$$
\begin{gathered}
\min _{\rho}: J=\mathbf{l}^{\top} \mathbf{u} \\
\text { s.t. }: \mathbf{K}(\boldsymbol{\rho}) \mathbf{u}=\mathbf{f} \\
\overline{\boldsymbol{\rho}}^{\boldsymbol{\top}} \mathbf{v} \leq V^{*} \\
\mathbf{0} \leq \boldsymbol{\rho} \leq \mathbf{1}
\end{gathered}
$$


where $\mathbf{l}$ is a vector with zeros and value one and minus one at the positions corresponding to the vertical displacement at the jaw corners. In order to save computational time only half of the design domain with symmetry boundary conditions is utilized in the computations. The gradients with respect to the projected densities are obtained using adjoint analysis and are given as

$$
\frac{\partial J}{\partial \overline{\boldsymbol{\rho}}_{j}}=-\boldsymbol{\lambda}^{\top} \frac{\partial \mathbf{K}}{\partial \overline{\boldsymbol{\rho}}_{j}} \mathbf{u}
$$

where $\boldsymbol{\lambda}$ is obtained as a solution to the following adjoint problem

$$
\mathbf{K}^{\top}(\rho) \lambda=1
$$

The gradients with respect to the design field are obtained by using the chain rule. The heat sink problem is defined in the same way as the minimum compliance problem with unit heat input uniformly distributed over the design domain.

The results for L-bracket optimization for the standard density based filter without Heaviside projection, and with Heaviside projections given by equations Equation 17 Equation 23 and Equation 21 are shown in Table 2 The modulus of elasticity for the solid material is taken to be one and the applied force is set to one as well. The density filter radius is $R_{f}=5.6 \mathrm{~L} / 200$. The volume fraction is $50 \%$. The discreteness of the obtained designs is represented with the following gray indicator [124]

$$
M_{n d}=\frac{\sum_{1}^{N} 4 \overline{\boldsymbol{\rho}}_{i}\left(1-\overline{\boldsymbol{\rho}}_{i}\right)}{N} \cdot 100 \%
$$

As expected the design obtained with density filter without any projection possesses large gray regions and actual realization would require post processing, i.e., removing the gray transition regions. The designs with Heaviside projections are with similar levels of intermediate design elements. Length scale on the solid regions for the designs obtained with Equation 17 and Equation 21 can be clearly identified. All solid features are larger than a dot with radius equal to the radius of the filter. On the other hand the void regions do not possess length scale, which is evident either from the sharp internal bracket corner or the sharp corners in the elements connections. Such sharp corners lead to stress concentration and are undesirable mechanical designs. The design obtained with the tanh projection does not have sharp internal bracket corner due to the extension of the design domain and the intermediate threshold $\eta=0.5$.

Heat sink designs are shown in Table 3 The volume fraction is $50 \%$ and filter parameters are the same as for the L-bracket optimization problem. It can be observed that all designs posses large gray regions with gray index in the same order as the gray index for the unprojected design. The projections cannot suppress the appearance of intermediate densities. Hence, the heat sink example is probably the simplest problem where new techniques for imposing length scale on black and white designs can be tested. 


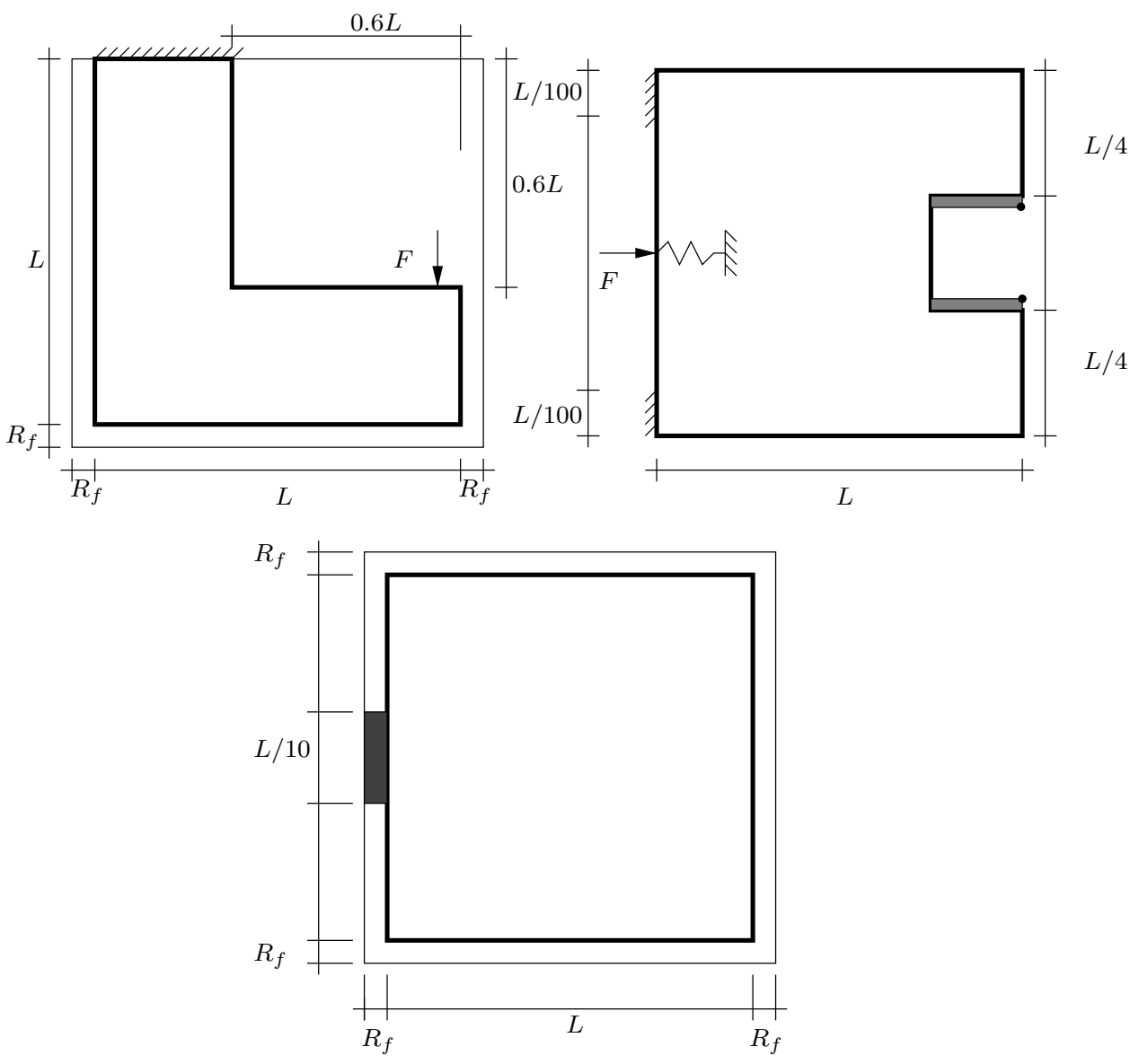

Fig. 1 Design domains and boundary conditions of: a) L-bracket, b) compliant mechanism, c) heat sink.

Results for compliant mechanism design are shown in Table 4 Only the upper half of the design domain shown in the table is utilized in the computations. The volume fraction is $30 \%$. The ratio of the output springs stiffness to the input spring stiffness is 0.005 . The output springs are vertical and are positioned at the output points shown with bold black dots in Figure 1 The thickness of the jaws is $0.05 \mathrm{~L}$. Similar to the L-bracket the projections decrease the gray level in the obtained designs. Length scale is clearly imposed on the solid regions for the design obtained with the Dilate and the Harmonic filters, however, some of the elements are with intermediate densities which again indicates that the projection alone cannot ensure zero/one designs. The void regions do not posses length scale which leads to the appearance of hinges in the connecting regions. 
Table 2 Single case designs for L-bracket with different filters and projections.

\begin{tabular}{ccccc}
\hline Filter & $\begin{array}{c}\text { Density filter } \\
\text { Equation 8 }\end{array}$ & $\begin{array}{c}\text { Dilate filter } \\
\text { Equation 17 }\end{array}$ & $\begin{array}{c}\text { Tanh filter } \\
\text { Equation 23 }\end{array}$ & $\begin{array}{c}\text { Harmonic filter } \\
\text { Equation 21 }\end{array}$ \\
\hline Obj. & 201.50 & 130.54 & 149.51 & 127.80 \\
\hline Gray $M_{n}$ & $20.06 \%$ & $2.63 \%$ & $0.93 \%$ & $1.10 \%$ \\
\hline
\end{tabular}

Table 3 Single case designs for Heat sink with different filters and projections.

\begin{tabular}{ccccc}
\hline Filter & $\begin{array}{c}\text { Density filter } \\
\text { Equation 8 }\end{array}$ & $\begin{array}{c}\text { Dilate filter } \\
\text { Equation 17 }\end{array}$ & $\begin{array}{c}\text { Tanh filter } \\
\text { Equation 23 }\end{array}$ & $\begin{array}{c}\text { Harmonic filter } \\
\text { Equation 21 }\end{array}$ \\
\hline Obj. & 133.43 & 126.77 & 116.52 & 125.97 \\
\hline Gray $M_{n}$ & $42.21 \%$ & $33.49 \%$ & $15.32 \%$ & $31.11 \%$ \\
\hline
\end{tabular}

In general the three field formulation can in some cases improve the discreteness of the obtained designs, however, for problems where the optimization can benefit from the appearance of gray regions, zero/one designs cannot be guaranteed. Heaviside projection with threshold projection $\eta=0$ or $\eta=1$ and filtered field obtained using filter function with finite support can ensure length scale either on the solid or the void regions in the design. However, the projections alone cannot provide length scale on both phases, therefore, they cannot prevent the appearance of sharp inter-element connections which lead to stress concentration. Furthermore, the appearance of sharp features either in solid or the void regions might lead to lack of manufacturability in some production processes due to lack of resolution. This is especially evident in compliant mechanism designs where small imperfections around the mechanism hinges can lead to completely disintegrated mechanisms. Manufacturability can be ensured either by taking into account the production uncertainties in the optimization process, or, in some cases by imposing length scale on both phases. 
Table 4 Single case designs for gripper with different filters and projections.

\begin{tabular}{ccccc}
\hline Filter & $\begin{array}{c}\text { Density filter } \\
\text { Equation 8 }\end{array}$ & $\begin{array}{c}\text { Dilate filter } \\
\text { Equation 17 }\end{array}$ & $\begin{array}{c}\text { Tanh filter } \\
\text { Equation 23 }\end{array}$ & $\begin{array}{c}\text { Harmonic filter } \\
\text { Equation 21 }\end{array}$ \\
\hline Obj. & 0.72 & 0.90 & 1.02 & 0.88 \\
\hline Gray $M_{n}$ & $31.49 \%$ & $12.25 \%$ & $2.10 \%$ & $5.53 \%$ \\
\hline
\end{tabular}

\section{On the similarities between three field representation and micro- and nano- scale production processes}

The three field representation provides the basic building block for controlling geometrical details of optimized designs. Two of the the three fields ( $\rho$ and $\widetilde{\rho}$ ) are seemingly pure mathematical entities. They are utilized in restricting the design space and removing the necessity of imposing additional constraints on the optimization problem. The original intent for introducing them in topology optimization was to ensure mesh independent solutions. However, due to the complex chain of transformations, often the link to the real physics of the problem is lost. Once the final design is obtained from the topology optimization process it is send for manufacturing. The designer usually post processes the optimized topology and sends it to the manufacturer who is responsible for transforming the design to a set of parameters for controlling the production machines. This transformation is based on purely geometric properties, i.e., the manufacturer minimizes the difference between the optimized topology and the one obtained by the manufacturing process by tuning the production parameters, e.g., 113. The design information which is lost during this transformation process may reduce the performance of the manufactured designs. Hence, avoiding or reducing the number of transformations will bring the performance of the realizations and the optimized design closer.

A striking similarity between micro- and nano-lithography production processes and the three field representation is demonstrated in [73. The article presents in details the link between electron beam lithography (EBL) and projection schemes in topology optimization and provides a direct map between the production parameters and the optimized topology. Later, a similar link is demonstrated to optical projection lithography in [165. The two processes and possible extensions to other micro- and nano- scale production technologies are presented in details in the following.

\subsection{Electron beam lithography}

Electron beam lithography 36 is a nano-fabrication process usually applied in small volume production. It allows direct writing of $2 \mathrm{D}$ design patterns down to sub $10 \mathrm{~nm}$ dimensions. The goal is to transfer a pattern to a thin layer of material 


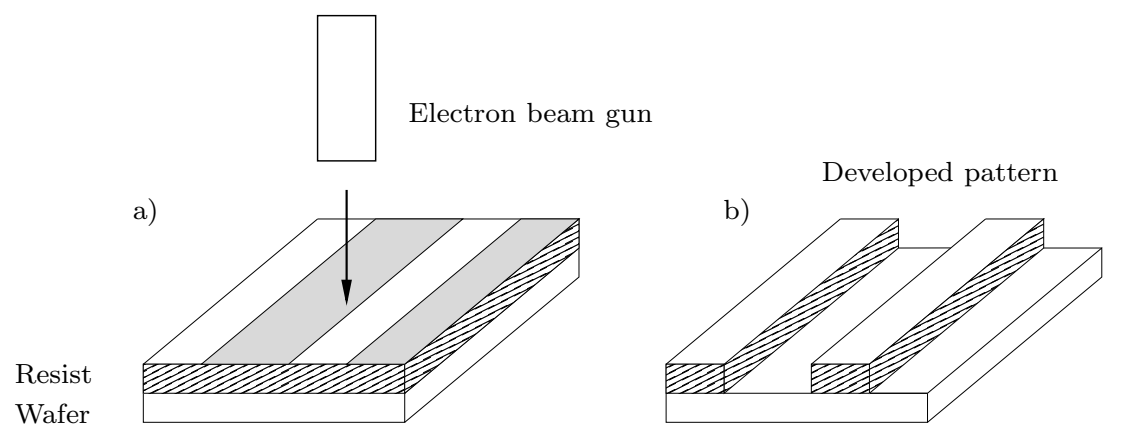

Fig. 2 Illustration of an E-beam lithography process in semiconductor microfabrication

referred to as resist in the literature. A typical setup, shown in Figure 2 consists of an electron beam which exposes the resist material laid on a substrate. The resist consists of a polymer which is sensitive to electron beam exposure [108].

The EBL process consists of two main phases. First, the resist is bombarded with electrons originating from the electron gun. The exposure causes local changes of the properties of the polymeric material. As a second step a solvent is added to the process in order to develop the exposed pattern on the resist. Two kinds of resists, negative and positive, are utilized in practice. Negative resist forms new bounds during the exposure, while for positive resist bounds are broken. The solvent removes molecules with smaller molecular weight which results in removing the exposed areas for positive resist and the unexposed for negative resits. The setup on Figure 2 demonstrates positive resist exposure and development. The process can be combined with other nano-production technologies for printing multimaterial or 3D layered designs 85, e.g., material deposition and subsequent etching. The focus here is only on demonstrating the link between the first two steps and the three field representation in topology optimization, hence, for more details the interested readers are referred to the existing literature on the subject, e.g., 85, 36.

In the exposure step the electron gun emits electrons which are directed by a magnetic and electrostatic field to a prescribed location on the resist [132]. The charge density directed to the resist is given as

$$
D(\mathbf{x})=T(\mathbf{x}) I
$$

where $T(\mathbf{x})$ denotes the time period for pointing the electron beam at position $\mathbf{x}$ and $I$ is the beam current. The exposure time can be specified for every point and is modeled as continuous variable. When an electron beam hits the resist, the electrons interact with the material which results in scattering of the electrons. Part of electrons reaching the substrate layer is reflected back causing a secondary backscattering to the resist. The forward and the backward scattering increases the width of the electron beam which is characterized by the so-called point spread 
function $F(\mathbf{x})$, which expresses the energy distribution of an incident point source due to scattering effects. The point spread function $D(\mathbf{x})$ is approximated by a sum of two Gaussians [29]. The final energy exposure $E(\mathbf{x})$ of the resists at point $\mathbf{x}$ is determined by a convolution integral

$$
E(\mathbf{x})=\int_{\mathbb{R}^{2}} F(\mathbf{x}-\mathbf{y}) D(\mathbf{y}) \mathrm{d} \mathbf{y}
$$

which resembles closely the density filtering process in topology optimization Equation 7

The development phase consists of several steps and can be modeled with different levels of complexity 40. The simplest model assumes constant threshold [26], i.e., for positive resist the material exposed to a level above a certain level $E_{\mathrm{cl}}$ is etched away. The level is controllable and depends on the process temperature, the solvent concentration, resist material, etc. The final resulting pattern written on the resist can be expressed as a Heaviside projection of the energy exposure

$$
P(\mathbf{x})=\mathrm{H}\left(E(\mathbf{x})-E_{\mathrm{cl}}\right)
$$

which coincides with the projection step in the three field density formulation. Hence, for EBL the three fields can be associated directly with real physical fields and the chain of transformation represents the different steps in the production process. The design field $\rho$ matches the exposure time, the filtered field $\widetilde{\rho}$ matches the energy exposure, and the density field $\widehat{\widetilde{\rho}}$ matches the final developed pattern. Such a map allows for direct transfer of the physical parameters to the manufactures which ensures manufacturability of the design and removes the post processing step. Furthermore, in addition to the optimization of the design performance, the map allows for optimization of the production parameters such as total exposure time and exposure patterns [74].

\subsection{Optical lithography}

Optical lithography similar to the EBL process aims at transferring a 2D pattern onto a thin layer of material. A typical production process consists of multiple steps 99. and here it is exemplified in Figure 3 A pattern is transferred onto a thin film layer deposited on a substrate. In a preparatory phase a thin layer of material is deposited on a wafer and then coated with light sensitive polymer called a photoresist. As a following step a mask (a pattern) is projected onto the photoresist with the help of an optical system. The projected image is developed and the exposed areas of the photoresist are removed. In the following steps the developed image is transferred to the film by an etching process and the photoresist is removed from the design.

The mask pattern can be represented using a binary image $I_{\mathrm{M}}(\mathbf{x})$ defined on a finite domain in $\mathbb{R}^{2}$. The intensity of the projected aerial image onto the photoresist is given as

$$
I_{A}(\mathbf{x})=\left|\left(I_{M} * H_{\beta}\right)(\mathbf{x})\right|^{2}
$$

where $*$ denotes the convolution operator and $H_{\beta}$ is the point spread function of the optical projection system. The etching phase is modeled with the help of the Heaviside projection and is given as

$$
I_{P}=H\left(I_{A}-\eta\right)
$$




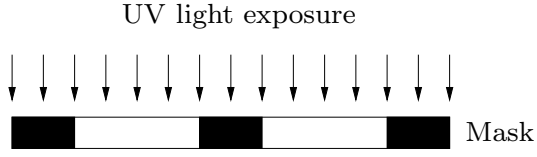

a)

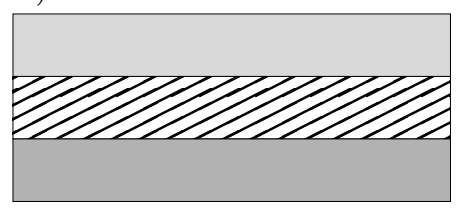

c)

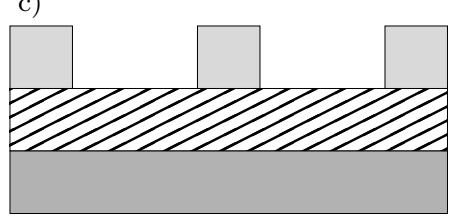

b)

Photoresist Film

Substrate

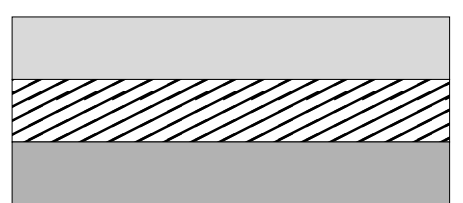

d)

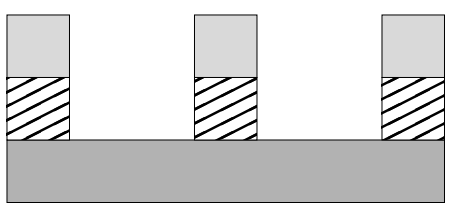

e)

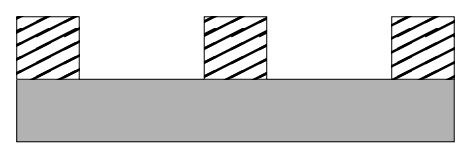

Fig. 3 Illustration of photo lithography process in semiconductor microfabrication: a) photoresist coated wafer, b) UV light exposure, c) photoresist development, d) etching, e) photoresist removal

where $I_{P}$ is the final developed pattern and $\eta$ represents an intensity threshold above which the material is etched away for positive photoresist. For a so-called negative photoresist the areas with intensity smaller than the threshold are etched away.

Similar to the EBL process presented in subsection 6.1 the three fields representation in topology optimization can be directly matched to the physical fields in photolitography [165]. The original design field $\rho$ can be utilized for representing the image mask $I_{M}$, the intermediate filtered field can represent the projected image and the developed design $I_{P}$ is represented by the projected field $\tilde{\widetilde{\rho}}$. The differences compared to the standard transformation chain $\rho \rightsquigarrow \widetilde{\rho} \rightsquigarrow \widehat{\widetilde{\rho}}$ are: 1) the filtered field is point wise squared, and 2) the mask $I_{M}$ is required to be a zero/one image. Detailed discussions and demonstration of the similarities can be found in [165.

6.3 Other micro- and nano-production processes

One of the main limitation of the EBL and photolitography production processes is the lack of capability for arbitrary three dimensional geometries. Three dimensional designs can be manufactured layer by layer by stacking several $2 \mathrm{D}$ 
projection-etching-deposition cycles on top of each other, however, the resulting process is still incapable of producing arbitrary 3D shapes. This limitation has stimulated research in mask less direct writing techniques 36 using focused optical beams. The light diffraction provides a limit on the resolution for direct writing, and an alternative based on non-linear interaction processes, the so-called two photon polymerization, has been proposed in [101. The technology has been developed further to produce features down to a few nanometers 51. The process utilizes ultra short laser pulses focused into the volume of a photoresistive material. A polymerization of the photoresist is initiated in the vicinity of the focal point, and after illumination of the 3D structure and subsequent etching, the polymerized regions will remain in the printed form, which allows fabrication of almost any complicated 3D pattern [80, 28. Due to the threshold behavior and the nonlinear nature of the process, a resolution beyond the diffraction limit can be realized [152. The three field representation model can be mapped directly to physical parameters by representing the focal point and the laser intensity with the design field, the exposed pattern with the filtered field and the final developed pattern with the projected field.

The research in nano-fabrication constantly provides new production processes capable of manufacturing devices with finer and finer resolution. The smallest features which can be produced are always limited by the available fabrication tools. On the other hand there are a large number of examples of extremely complex structures assembled on molecular level with sub-nanometer scale features. Such patterns in nature are realized by self assembly which provides one of the most promising mechanism for producing devices in the sub-nanometer range [85]. The process can be modeled numerically and optimized template patterns have been demonstrated in [1]. Similarities with the three field representation can be easily identified, however, topology optimization of a full device still remains to be demonstrated.

The above examples demonstrate the flexibility of the three field model to represent real physical fields in standard macro- and nano- manufacturing processes. Topology optimization based on this model controls directly the input production parameters and results in designs which incorporate the physical limitations in the design process. Optimization driven directly with the physical parameters avoid the post processing steps known as proximity effect correction in electron beam lithography and optical proximity correction in photolithography, and result in manufacturable topologies.

\section{Topology optimization under geometrical uncertainties}

Uncertainties in the production parameters are inevitable parts of every manufacturing process. Therefore, their inclusion in the optimization is necessary in order to ensure manufacturable designs with robust performance. The three field representation provides a excellent base for integrating the uncertainties in the topology optimization process and this application has been demonstrated in a number of problems.

Replacing the threshold parameter $\eta$ in the projection step with a random variable $\eta \in \mathcal{P}$, where $\mathcal{P}$ is a selected random distribution, can be utilized for modeling uniform erosion and dilation along the design perimeter [90]. The pro- 
jection step represents the etching process in lithography, therefore, the above modification can represent uncertainties in the temperature or the chemical concentration which leads to different threshold values. The deterministic objective function in Equation 1 can be replaced with a combination of the mean deterministic objective function and its standard deviation $\mathrm{E}(J)+\kappa \mathrm{STD}(J)$ [100, where, $\kappa$ is free parameter selected by the designer. The above modification tries to improve the average performance and at the same time to reduce its variability. The main difficulties with such a reformulation is the selection of the parameter $\kappa$ and the fact that such an objective is not a coherent risk measure [120, i.e., if a design $\rho_{1}$ is outperforming design $\rho_{2}$ for almost all possible realizations the above objective might not reflect this property. Nevertheless the above reformulation has been demonstrated to produce robust designs for a number of problems in mechanics [115] and electromagnetics [4].

The constant threshold model can model only uniform erosion and dilation along the design perimeter. An extension has been proposed in [122, where the random variable has been replaced with a random field defined over the design domain. In this way spatially varying geometric imperfections can be easily represented. Similar models for level set approaches have been proposed in 30. and 31. An additional study including spatially varying uncertainties in the material parameters is presented in [91]. One of the main conclusions is that spatially varying geometric uncertainties have very little effect on the topology of the optimized designs and often neglecting the spatial variability and considering constant threshold along the design domain produced results with similar performance, especially for mechanical design problems such as minimum compliance and compliant mechanisms. Recently it has been demonstrated in 32 that spatial variations can affect severely the topology for wave propagation problems. An alternative model has been proposed in 74 where the manufacturing uncertainties have been modeled as misplacement of material. Such a model can be utilized in the 3D printing processes where material is deposited by the printer head layer by layer.

The computational cost in the case of stochastic variations increases significantly and depends on the dimensionality of the stochastic space. In 122 Monte Carlo (MC) sampling has been utilized for obtaining the mean and the standard deviation of the system response. Even though MC possesses dimension independent convergence, its convergence speed is relatively slow [157. An alternative stochastic collocation sampling approach has been investigated in [90, 91] and 44. The main advantage is that the already developed deterministic solvers are utilized without any modifications in the optimization process. However, for fast oscillatory uncertainties and small number of sampling points without any error control the optimization process often tunes the design for the sampling points and any intermediate realizations may possess responses which are not robust with respect to the true non-discrete realizations [44. A topology optimization formulation with error control on the mean and the standard deviation remains to be demonstrated.

In attempt to decrease the computational cost perturbation based stochastic solvers have been utilized in 92. It has been clearly demonstrated that in general the Taylor expansion of the response around a single point in the stochastic space cannot capture the overall behavior and might lead to the same effects observed in 44, i.e., tuning the design performance for the expansion point. However, for relatively smooth problems it has been demonstrated in [75] that the perturbation 
approach can represent the response of the system due to stochastic imperfections and can lead to significant reduction of the computation time for geometrically non-linear optimization problems.

Another way to account for the manufacturing and exploitation uncertainties is to utilize the so-called reliability based design optimization (RBDO) [102]. For general discussion, overview and comparison between the different approaches the reader is referred to [50, 21, [46] and [102]. In RBDO the objective is either to minimize the probability of failure subjected to a set of additional constraints or the probability of failure is set as a constraint to the optimization problem. The probability of failure is often estimated approximately using the so-called first order reliability method (FORM) or second order reliability method (SORM). Such approaches rely on the stochastic behavior around a single point in the stochastic space (most probable failure point MPP) and suffer from the same disadvantages in optimization as the the perturbation approaches, i.e., the design is optimized for a particular point (set of parameters) in the stochastic space and some of the more important regions might be omitted by the optimizer. Reliability based topology optimization has been demonstrated for compliant mechanisms in [103] and for minimum compliance in 81 .

The above approaches are non-intrusive, i.e., one can utilize standard deterministic solvers for evaluating the moments of the response. For stochastic perturbation approaches only slight modifications are necessary. Another alternative for estimating the stochastic response is the stochastic Galerkin approach [55]. The method requires development of new solvers and for large dimensionality in the stochastic space might result in extremely large systems of equations. In topology optimization the method has been utilized in minimum compliance optimization under material uncertainties 138 .

A large number of research papers are devoted to the exploitation of uncertainties like random inputs or imperfections in boundary conditions, e.g., 83, 98, 43] and [162. It should be emphasized that the the focus here is on manufacturability and length scale of the the optimized designs, therefore, a detailed review on topology optimization under other types of uncertainties is omitted here.

Often the exact distribution of the random variables is unknown and it is desirable to account only for the worst case scenario from all possible design realizations. Such an approach leads to the so-called min / max formulation which in the case of minimum compliance optimization and compliant mechanism designs is given as

$$
\begin{gathered}
\min _{\boldsymbol{\rho}}: \max _{\eta} J(\boldsymbol{\rho}, \mathbf{u}, \boldsymbol{\eta}) \\
\text { s.t. }: \mathbf{K}\left(\boldsymbol{\rho}, \boldsymbol{\eta}^{*}\right) \mathbf{u}=\mathbf{f} \\
\max _{\eta} V(\boldsymbol{\rho}, \boldsymbol{\eta}) \leq V^{*} \\
\mathbf{0} \leq \boldsymbol{\rho} \leq \mathbf{1}
\end{gathered}
$$

where $\boldsymbol{\eta}$ is a vector with all random parameters and $\boldsymbol{\eta}^{*}=\arg \max _{\eta} J(\boldsymbol{\rho}, \mathbf{u}, \boldsymbol{\eta})$. The volume constraint $\max _{\eta} V(\boldsymbol{\rho}, \boldsymbol{\eta}) \leq V^{*}$ can also be replaced with a fixed point in the random space. Such a formulation is demonstrated in 147] where the volume constraint is applied on the most dilated design which actually coincides with the $\arg \max _{\eta} V(\boldsymbol{\rho}, \boldsymbol{\eta})$. Alternatively the volume constraint can be applied on the mean volume 122. 
For compliant mechanism design and threshold projection $\eta \in\left[\eta_{d}, \eta_{e}\right]$, which is constant in the design domain, it has been observed that the worst performing design is either the most eroded or the most dilated design [125]. This has been utilized in [147], 115] for simplifying the formulation given by Equation 45 to

$$
\begin{aligned}
\min _{\boldsymbol{\rho}}: & \max \left\{J_{e}, J_{d}\right\} \\
\text { s.t. } & : \mathbf{K}\left(\boldsymbol{\rho}, \eta_{d}\right) \mathbf{u}_{d}=\mathbf{f} \\
& \mathbf{K}\left(\boldsymbol{\rho}, \eta_{e}\right) \mathbf{u}_{e}=\mathbf{f} \\
& V\left(\boldsymbol{\rho}, \eta_{d}\right) \leq V^{*} \\
& \mathbf{0} \leq \boldsymbol{\rho} \leq \mathbf{1}
\end{aligned}
$$

where $\left\{J_{e}, J_{d}\right\}$ are standard deterministic objectives for thresholds $\eta_{e}$ and $\eta_{d}$, respectively. As the worst performing case is either for threshold $\eta_{e}$, or threshold $\eta_{d}$, the case for $\eta_{i}$ corresponding to the blueprint design supplied to the manufacturer, can be omitted in the optimization formulation which can save around a third of the computational time compared to the formulation in [147. In contrast to the deterministic case the robust formulation requires one or more systems solutions corresponding to each realization which significantly increases the computational load.

In the general case the worst case is not known in advance and a large number of samples in the stochastic space is utilized in order to approximate the random response and to find the worst case scenario. The optimization formulation for such a case becomes

$$
\begin{aligned}
\min _{\boldsymbol{\rho}} & : \max \left\{J_{i}, i=1 \ldots N_{s}\right\} \\
\text { s.t. } & : r\left(\rho, u, \boldsymbol{\eta}_{i}\right)=0, \quad u \in \mathcal{U}_{\mathrm{ad}}, i=1 \ldots N_{s} \\
& g_{i}\left(\rho, u, \boldsymbol{\eta}_{i}\right) \leq 0, \quad i=1 \ldots N_{g}, i=1 \ldots N_{s} \\
& \rho \in \mathcal{D}_{\mathrm{ad}}
\end{aligned}
$$

where $N_{s}$ is the number of samples and $\boldsymbol{\eta}_{i}$ is a point in the random space. The above formulation has been utilized for photonic crystal design [145] and 146], for topology optimization in acoustics [32, and for material design in [149]. Without any constraints min / max formulations have been demonstrated for topology optimization in photonics in 116 and 117 where a cheap surrogate model has been utilized for reduction of the computational cost. Another demonstration in photonic crystal design considering manufacturing uncertainties based on robust regularization of a function is shown in [105]. In compliant mechanism design and minimum compliance problems a multiple load case scenario with localized damage has been demonstrated to limit the maximum member size in [76].

The max function is not differentiable and the optimization problem given by Equation 38 is usually reformulated using the so-called bound formulation as

$$
\begin{aligned}
\min _{\boldsymbol{\rho}} & : z \\
\text { s.t. } & : J_{i} \leq z, \quad i=1 \ldots N_{s} \\
& r\left(\rho, u, \boldsymbol{\eta}_{i}\right)=0, \quad u \in \mathcal{U}_{\mathrm{ad}}, i=1 \ldots N_{s} \\
& g_{i}\left(\rho, u, \boldsymbol{\eta}_{i}\right) \leq 0, \quad i=1 \ldots N_{g}, i=1 \ldots N_{s} \\
& \rho \in \mathcal{D}_{\mathrm{ad}}
\end{aligned}
$$


The above formulation can be naturally implemented using any MMA implementation [133.

Replacing the deterministic optimization formulation with a formulation which accounts for uncertainties in the production process removes the human factor in the optimization process. First the three field model ensures that the design is manufacturable and second requiring robustness of the response ensures that the obtained designs will posses robust behavior for a wide range of variations in production parameters. It has been observed in [147, [122, 93 that requiring robustness of the performance with respect to uniform erosion and dilation for compliant mechanism design removes the single node hinge problem, demonstrated in Table 7 and results in black and white design with minimum length scale on the obtained topology. It should be stressed that the length scale is guaranteed only if the designed topology does not change for all possible realizations. Such changes in topology have been demonstrated for photonic crystals in [145], for optimization in acoustics in 32 and for compliant mechanisms in 88 . Nevertheless the designs obtained by requiring robustness of the performance can be send to manufacturing without any post processing and human interventions. The full cycle has been demonstrated in the design of auxetic materials in 14 .

Examples of designs with robust performance with respect to uniform erosion/dilation $\eta \in[0.35,0.65]$ for the L-bracket, the Heat sink and the compliant gripper optimization problems are shown in Table 5 Table 6 and Table 7 respectively. In the first row of each table the most eroded and the most dilated designs are obtained using the Harmonic mean filter given by Equation 21 and Equation 22 respectively. The filter radius for the Harmonic filter is set to $2.17 L / 200$. As it can be observed, such a model does not impose length scale, cannot ensure black and white design, and cannot prevent hinges in compliant mechanism designs. This is due to the fact that the Harmonic erode and dilate operations can produce projected fields with equal values, and hence, they cannot model erosion/dilation imperfections. In contrast the tanh projection given by Equation 23 always produces different projected field values for different thresholds. This property results in length scale on both the solid and void regions and in designs close to black and white.

\subsection{Minimum compliance design with random projection threshold}

The main reason that the robust formulation has not yet been adopted by the industry is the additional computational cost associated with solving the multiple design realizations and the large number of optimization iterations due to the continuation strategy. The most popular topology optimization problem is minimum compliance design. By careful analysis the computational cost for the robust formulation presented above can be reduced to be equal to the cost associated with solving a single case deterministic problem. 
Table 5 L-bracket robust design (dilated, intermediate and eroded design realizations).

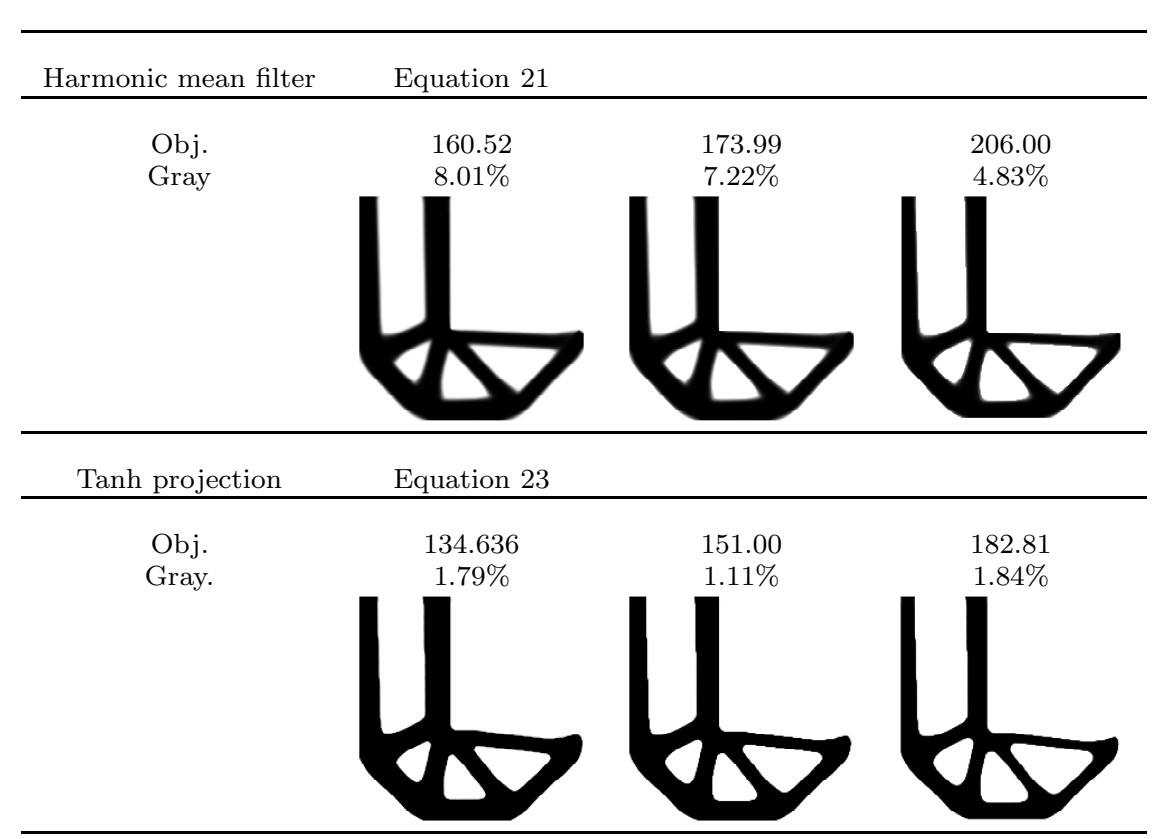

The threshold projection is assumed to be uniform and the threshold is modeled as a random variable $\eta \in \mathcal{P}\left(\eta_{d}, \eta_{e}\right)$, where $\mathcal{P}$ is an unspecified random distribution with support $\left[\eta_{d}, \eta_{e}\right]$. The compliance is given as

$$
J(\rho, \eta)=\int_{\Omega} \mathbf{f}(\mathbf{x}) \mathbf{u}(\mathbf{x}) \mathrm{d} \mathbf{x}
$$

where $\mathbf{u}$ depends on the physical density and on the threshold $\eta$ though the state equation. The compliance is positive and for $E_{j}(\mathbf{x}) \geq E_{i}(\mathbf{x}), \forall \mathbf{x} \in \Omega$ the compliance $J_{j}$ is always smaller or equal to the compliance $J_{i}$, i.e. $J_{j} \leq J_{i}$. Using the SIMP model given by Equation 5 with a physical density field obtained by projecting filtered density field $\widetilde{\rho}$ with thresholds $\eta_{i}$ and $\eta_{j}$, the following relation can be identified

$$
E_{j}\left(\mathbf{x}, \bar{\rho}_{j}\right) \geq E_{i}\left(\mathbf{x}, \bar{\rho}_{i}\right), \quad \forall \eta_{j} \leq \eta_{i}
$$

where $\bar{\rho}_{k}=\mathrm{H}_{\eta_{k}}(\widetilde{\rho}), k=i, j$. Therefore, the maximum compliance for any filtered density distribution $\widetilde{\rho}$ is obtained for the most eroded realization $\eta_{e}$, i.e.

$$
\eta_{e}=\arg \max _{\eta \in \mathcal{P}\left(\eta_{d}, \eta_{e}\right)}\{J(\rho, \eta)\}
$$

Using similar argumentation the maximum material volume $\max _{\eta \in \mathcal{P}\left(\eta_{d}, \eta_{e}\right)}\{V(\rho, \eta)\}$ can be obtained for the most dilated design, i.e.

$$
\eta_{d}=\arg \max _{\eta \in \mathcal{P}\left(\eta_{d}, \eta_{e}\right)}\{V(\rho, \eta)\}
$$


Table 6 Robust design for Heat Sink (dilated, intermediate and eroded design realizations).

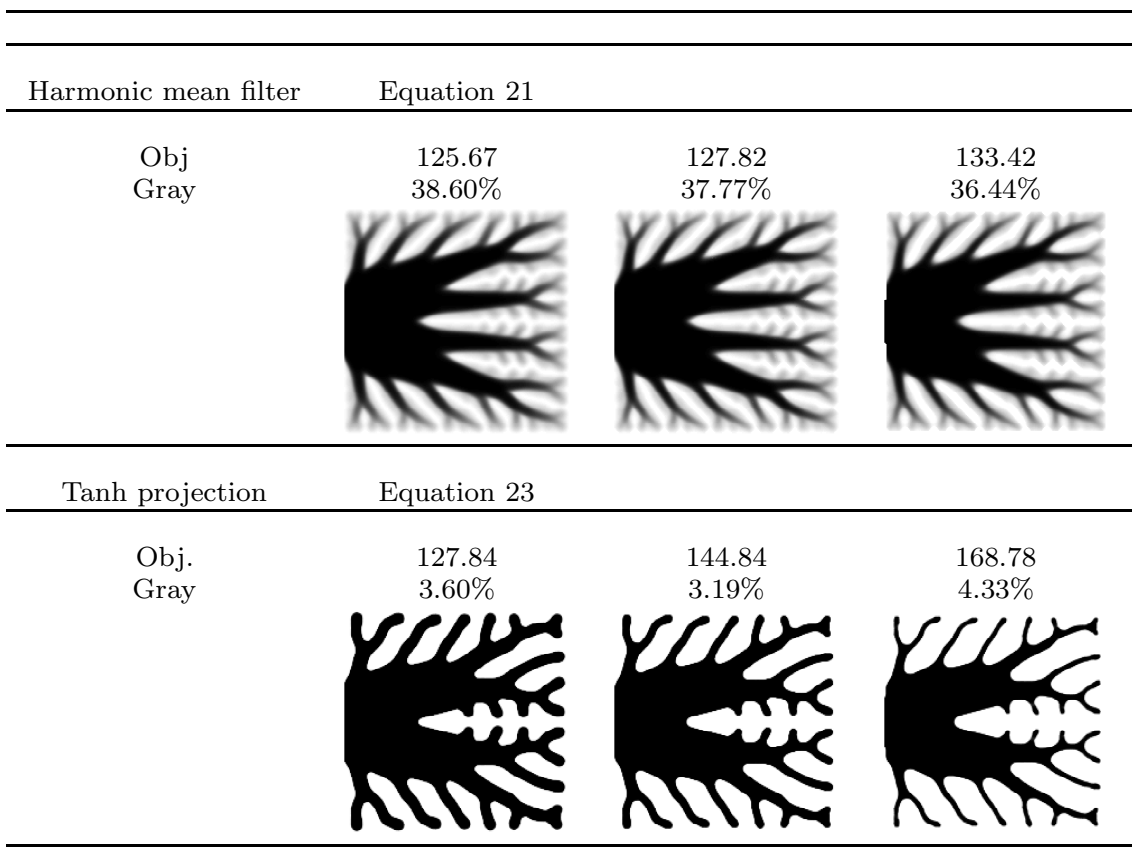

Table 7 Robust design for Gripper (dilated, intermediate and eroded design realizations).

\begin{tabular}{|c|c|c|c|}
\hline Harmonic mean filter & Equation 21 & & \\
\hline $\begin{array}{l}\text { Obj. } \\
\text { Gray }\end{array}$ & $\begin{array}{c}0.88 \\
12.73 \%\end{array}$ & $\begin{array}{c}0.91 \\
11.88 \%\end{array}$ & $\begin{array}{c}0.88 \\
9.54 \%\end{array}$ \\
\hline Tanh projection & Equation 23 & & \\
\hline $\begin{array}{l}\text { Obj. } \\
\text { Gray }\end{array}$ & $\begin{array}{c}0.85 \\
2.57 \%\end{array}$ & $\begin{array}{c}0.91 \\
2.32 \%\end{array}$ & $\begin{array}{c}0.85 \\
2.94 \%\end{array}$ \\
\hline
\end{tabular}

where the material volume is computed as

$$
V(\rho, \eta)=\int_{\Omega} H_{\eta}(\widetilde{\rho}) \mathrm{d} \mathbf{x}
$$


Therefore, the optimization formulation given by Equation 45 can be replaced with an equivalent one given as

$$
\begin{gathered}
\min _{\boldsymbol{\rho}}: J\left(\boldsymbol{\rho}, \mathbf{u}, \eta_{e}\right)=\mathbf{f}^{\top} \mathbf{u} \\
\text { s.t. }: \mathbf{K}\left(\boldsymbol{\rho}, \eta_{e}\right) \mathbf{u}=\mathbf{f} \\
\\
V\left(\boldsymbol{\rho}, \eta_{d}\right) \leq V^{*} \\
\mathbf{0} \leq \boldsymbol{\rho} \leq \mathbf{1}
\end{gathered}
$$

In contrast to the general case the reduced formulation requires only a single state solution. Furthermore, as the volume constraint is always active and $\boldsymbol{\rho}_{d}>\boldsymbol{\rho}_{e}$, the problem is self penalizing and the penalty parameter in the SIMP interpolation can be set to 1 , i.e, the interpolation between void and solid is linear. The above formulation leads to black and white designs with response robust with respect to uniform erosion and dilation along the design perimeter. Due to the lack of local minima and maxima between the most eroded and the most dilated case it results in clearly defined minimum length scale on both the solid and the void regions for a blueprint design obtained for an intermediate $\eta$. The same reduction can be applied to any spatially varying projection threshold which is bounded between two constant values, $\eta_{d} \leq \eta(\mathbf{x}) \leq \eta_{e}$. Hence the worst case formulation in minimum compliance topology optimization leads to a problem with computation cost similar to the computational cost for the deterministic minimum compliance optimization problem.

The methodology is demonstrated in Table 8 and Table 9 for L-bracket and heat sink designs. The boundary conditions, the filter radius and the input to the system is the same as for the robust optimization case. The formulation results in black and white designs with minimum length scale imposed on both the solid and the void regions. The objective for penalization parameter $p=1$ is slightly better for both of the considered cases. Using linear interpolation decreases the number of local minima and maxima for the optimization problem. Based on the authors experience linear interpolation improves the convergence speed. Different length scales can be imposed on the void and the solid regions by selecting thresholds which are not symmetric with respect to the intermediate threshold $\eta=0.5$ [147]. Using such an approach the designers can control the rounding radius and hence the stress concentration in the final designs without explicitly accounting for stress concentration in the optimization problem.

\section{Length scale in macro scale production processes}

Manufacturing is the technological process which utilizes different physical processes to modify the geometry and properties of a given amount of material in order to create parts of products as well as to assemble these parts in a final product [58]. The process consists of a sequence of operations which brings an initial block of material closer to the desired final detail. The set of possible operations can include wide ranges of sub-processes like, casting and molding, material 
Table 8 L-bracket robust design using single realization

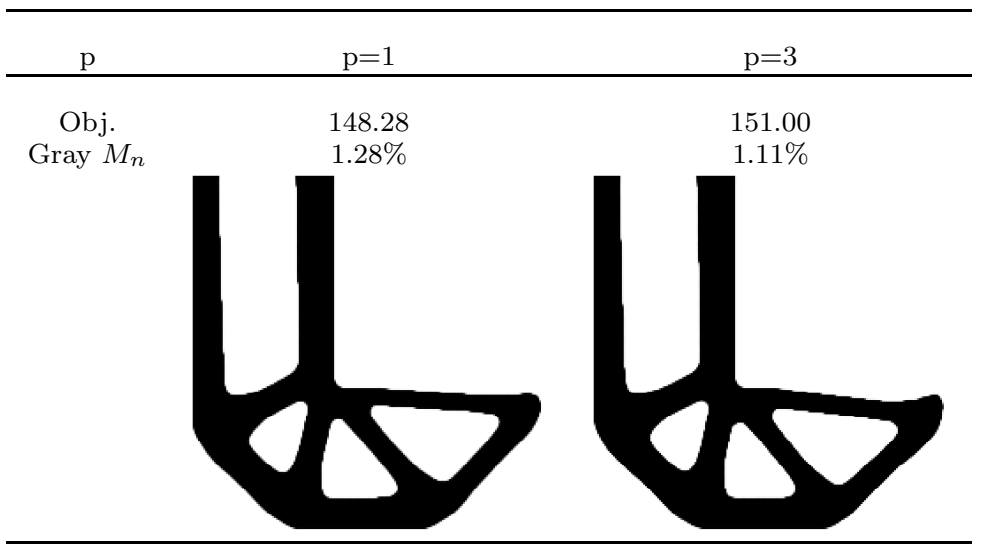

Table 9 Heat sink robust design using single realization

\begin{tabular}{ccc}
\hline $\mathrm{p}$ & $\mathrm{p}=1$ & $\mathrm{p}=3$ \\
\hline $\mathrm{Obj}$. & 135.69 & 144.8662 \\
\hline $\mathrm{Gray} M_{n}$ & $3.08 \%$ & $3.20 \%$ \\
\hline & &
\end{tabular}

forming, material removal processes (turning, drilling, milling, grinding), material deposition processes, etc. Each one of these technological operations imposes some restrictions on the parts under production. Therefore, in order to ensure manufacturability of designs obtained using topology optimization these restrictions need to be imposed during the optimization process.

Several articles have presented such methodologies in topology optimization. In casting one of the important requirements is that the casting molds should be removable without damaging the cast part and the mold tools [154, i.e., the mold parts should not have concave geometry and any interior voids. The first mathematical formulation of casting constraints was proposed in 164. Topology optimization using the constraints has been demonstrated in 72 . Transformation of the constraints making them applicable to level set-based approaches are demonstrated in [154] and [155]. For the density based formulation, [54] has pro- 
posed an explicit parametrization for casting constraints based on a Heaviside projection which recently has been extended in 97 . An alternative is proposed in 67] where projection based algorithms have been utilized for imposing casting as well as milling constraints on topology optimized designs. A general overview for applications in the aerospace industry can be found in [170] where the main focus is on the design of thin walled structures with stiffeners [169]. For laminated composites, an optimization formulation can be found in [129. A machining feature based level set optimization approach is presented in 96. Most of the casting and machining manufacturing constraints do not affect explicitly the length scale imposed on the solid regions of the design. They are related mainly to the convexity of the design envelope and the length scale imposed on the void regions. Therefore, the minimum length scale on the solid is governed by the manufacturing uncertainties, i.e., the same considerations applied to micro- and nano- scale production processes are applicable to the manufacturing processes mentioned above.

Often a number of features with explicit shape are required to be embedded in the optimized design due to aesthetic considerations in architecture, manufacturing restrictions, or holes and components passing through the design 33 . Most of the publications presenting such optimization formulations utilize level set approaches, e.g., 104, 168. A general review can be found in 160 where a large number of density based examples are presented. Requiring embedded design features does not affect the length scale on the actual free design domain and additional constraints based either on the requirement for robustness or explicit length scale are discussed later in this section.

Additive manufacturing (AM), also known as 3D printing, is a relatively new production technology. In contrast to traditional machining where the manufacturing relies on material removal, AM is based on adding material layer by layer, thus, avoiding large part of the manufacturing constraints imposed by other production techniques. Often a design obtained by a CAD system can be directly fabricated without the need of process planning 56. Different 3D printing processes have been developed for different materials. Modern devices can utilize polymers, metals, ceramic, and even bio-materials for printing human tissues, e.g., 141, 139, 42. Due to the fast development of the technology, cheap desktop 3D printers are widely available for both prototyping, as well as mass production. However, even though AM has received big impulse both from industrial manufacturers and hobby enthusiasts many challenges still exist and are subject to active research. Several of them outlined in 112 and related to the current review paper are the lack of computationally efficient 3D topology optimization software, the lack of robust modelling and optimization tools utilizing material microstructures, and the need for post processing of optimized designs.

Topology optimization as a design process is the perfect supplement to the additive manufacturing as it can completely utilize the manufacturing freedom. Topology optimization for additive manufacturing has been demonstrated in a large number of articles [25], [137] and recently for multi material designs in [52, 136. One of the issues is the post processing step for designs obtained using pure SIMP approach, i.e., the physical density is modeled using the filtered design field and posses gray regions. Such a post processing step can be removed ether by requiring robustness of the design performance with respect to variations in the geometry or by obtaining black and white design with clearly defined minimum length scale. The first approach relies on the theory presented in section 7 and is 
demonstrated for auxetic material microstructure design in 14. The second will be discussed in details later in this section.

Even though additive manufacturing avoids most of the design constraints, the process requires that the manufactured structures are self supported. This requirement can be fulfilled by constraining the size of the overhanging part. The approach presented in 95] is essentially a post processing step and as discussed earlier might destroy the optimality of the solution. A solution based on projections without additional constraints is presented in [53.

In order to save material and increase the production speed in 3D printing the production details are printed using thin walls for the surface and internal porous filling material. Such a process imposes several challenges for topology optimization. The first one is resolving the thin wall and the internal porous structure and the second is parametrization of the different parts of detail during the optimization process. A parametrization based on the standard three field representation combined with an additional field utilizing the gradient of the physical density is presented in 34. In such a parametrization the filling material can be realized using uniform material microstructure, thus avoiding the requirement for detailed modeling inside the design. This microstructure can be designed using topology optimization applied for material design where additional local constraints can be easily imposed [65]. Uniform material microstructure does not utilize the additional freedom provided by the technology, i.e., the material properties can vary spatially within the manufacturing domain. A non-uniform microstructure can be designed using hierarchical homogenization 118, 35. The varying microstructural cells lack connectivity which requires modification of the design for connecting the different microstructures. Furthermore, the homogenization theory does not account for the boundary conditions and local effects (concentrated loading, loss of local stability). An alternative which provides spatially varying manufacturable microstructures without any post processing is presented in [4] and [3]. The presented methodology resolves all microstructural details and accounts for boundary conditions and localized effects.

\subsection{Minimum length scale}

With the exception of compliance like optimization problems the robust topology optimization formulation requires the solution of several state problems. The number of state problems depends on the ratio of the characteristic length of the design domain and the correlation length of the stochastic uncertainties. It can vary from several [147, 90. to several hundred and thousand solves [4] and [76]. The increased computational cost is often unacceptable especially for problems where the solution of a single state problem is expensive. Therefore, an alternative solution is to impose a minimum length scale with the assumption that such an additional constraint will provide at least to some extend robustness of the performance with respect to uncertainties in the production process.

The first article 61] to propose an approach for imposing length scale on the solid regions in the design domain utilizes the three field design representation with density field obtained using filtering with finite support. The filter support provides the smallest building block for the phase which can be viewed as a union of an infinite number of filter support shapes. The projection threshold is set to 
$\eta=0$ for imposing length scale on the solid phase and $\eta=1$ for imposing length scale on the void phase.

Imposing length scale only on one of the phases does not always guarantees manufacturability [147], e.g., hinges cannot be avoided in mechanism design Table 4 and the appearance of gray transition regions cannot be avoided Table 3 Therefore, an explicit length scale control on both phases is necessary. Such a mathematically rigorous approach has been proposed recently for density based topology optimization in [166.

For density based topology optimization the minimum length scale on both phases is imposed with the help of an additional constraints [166. The idea is based on the observation in 147, i.e., a length scale can be guaranteed on both phases for the intermediate design if the topology does not change for all possible design realizations. It should be pointed out that in case of standard robust topology optimization formulation this feature is a result from the optimization and is not guaranteed for all optimized designs. In the methodology proposed in 166 it is explicitly required, hence, it is guaranteed that the final design will posses minimum length scale.

A topology described by a continuous density field does not change if the following two conditions are satisfied

$$
\begin{array}{ll}
\widetilde{\rho}(\mathbf{x}) \geq \eta_{e}, & \forall \mathbf{x} \in \Omega_{1}=\{\mathbf{y} \in \Omega \mid \bar{\rho}(\mathbf{y})=1, \text { and } \nabla \widetilde{\rho}=0\} \\
\widetilde{\rho}(\mathbf{x}) \leq \eta_{d}, & \forall \mathbf{x} \in \Omega_{2}=\{\mathbf{y} \in \Omega \mid \bar{\rho}(\mathbf{y})=0, \text { and } \nabla \widetilde{\rho}=0\}
\end{array}
$$

The above conditions utilize the three field formulation. The first condition ensures minimum length scale on the solid phase and the second condition ensures minimum length scale on the void phase. The numerical implementation is discussed in details in [166] and an example for heat sink design with imposed minimum length scale on both phases is shown in Table 10 An approach which also is based on an additional constraint is proposed in [114. This alternative requires more complex implementation however.

For level set approaches, mathematically rigorous formulations for imposing minimum length scale have been proposed in [106] and [6]. Attempting to provide simpler formulations several other papers 68, 161, and [153 propose a skeleton based idea to control the minimum length scale. The idea is to extract the medial zone of a structure and to constrain the corresponding density values. A shortcoming of the presented formulations is that the gradients of the medial zone are neglected. The same is valid for the extension to density based topology optimization proposed in 161. The possible shortcomings for these approaches are discussed in 6.

As stated in [6], so far the perfect formulation for imposing minimum length scale remains to be discovered. Several critical cases for the level set formulation are discussed in [6. For the density based formulation presented in [166], the requirement is that the constraints are applied on some initial topology. As demonstrated in [166] the topology can be changed by the optimization process. A comparison [166] to compliant mechanisms optimized using the robust formulation shows that for the intermediate blueprint design the performance is slightly better than the robust design, however, for different realizations the variations in the performance are significantly larger for the mechanism optimized only for minimum length scale. The same effect is observed for photonic crystals in [166]. 
Table 10 Heat sink design with imposed minimum length scale based on additional constraints given by Equation 46 and Equation 47

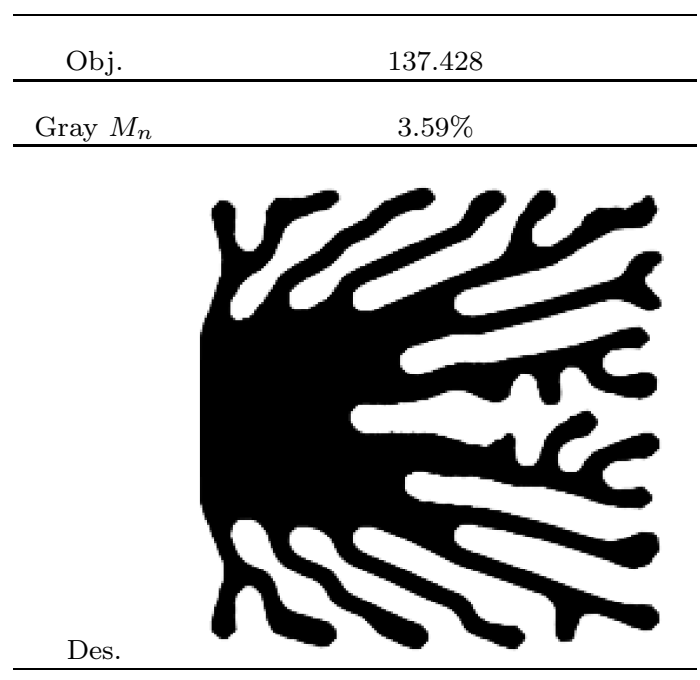

\subsection{Maximum length scale}

Imposing restrictions on the maximum length scale of the void phase, the solid phase, or of both phases result in the appearance of redundant members which provide diversification of the load path [59]. This effect can be clearly observed in [76] where maximum length scale on the design members appears as a result of the required robustness with respect to localized damage. Maximum length scale on optimized designs can be required due to technological limitations, e.g., for high rise buildings, the size of the bottom columns is limited due to difficulties with transportation and lifting, or due to limitations in the maximal size of the available steel sheets. Other maximum length scale requirements may be imposed due to desired properties which cannot be included in the optimization process. An example is requiring a specific porosity in scaffold designs which can be indirectly imposed by setting constraints on the maximum length scale.

In 59] maximum length scale is enforced on topology optimized designs by restricting the amount of material in the neighborhood of each point in the design domain. This approach leads to a large number of constraints (one constraint for each design variable). Two alternatives are proposed in 88. The first one is based on the design of band pass filters and is an extension of the idea presented in 82 . Band pass filters can be designed directly in the frequency domain and the filtering can be realized using $\mathrm{FFT} / \mathrm{iFFT}$ transformations. An alternative is to present the filtered field as the difference of two filtered fields obtained from the same density field $\rho$, i.e., one obtained with larger filter radius and the other obtained with smaller filter radius 88 . The Fourier transform of the resulting field does not posses any values around the zero frequency which results in suppression of the appearance of large void or solid areas in the filtered design. The proposed technique restricts the design space and therefore does not require any additional 

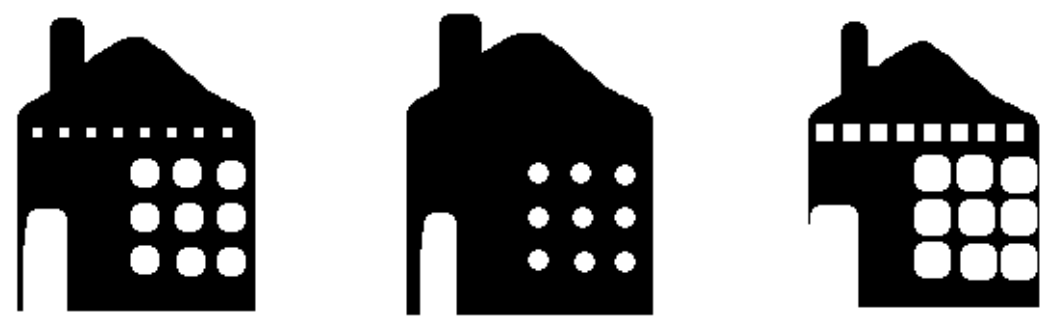

Fig. 4 Illustration of erosion and dilation morphological operations (left: original image, right: eroded image, middle: dilated image).

constraints. The main limitation is that the maximum length scale is defined only loosely in the frequency domain.

The second alternative proposed in 88 is based on morphological operators [109]. A figure of a house together with two images obtained by dilation and erosion operations are shown in Figure 4 The erosion and the dilation operations are applied with a rectangular box with dimensions $5 \times 5$ pixels. As demonstrated all solid(black) elements with dimensions smaller than the structural element $(5 \times 5$ box) are erased in the erosion operation and all void elements with dimensions smaller than the structural element are erased in the dilation operation. Hence, this behavior can be utilized in defining maximum length scale in topology optimization. The maximum length scale is defined with the help of a structural element which can take any shape, i.e., maximum length scale on a phase is defined by the parameter/parameters necessary to define the smallest structural element with specified shape for which the eroded/dilated design coincides with the design domain filled entirely with the other phase. If an erosion operation with a specified structural element is applied on a design and the result is a design domain filled with void phase, the maximal feature in the solid phase is smaller than the specified structural element. If a dilation operation is applied on a design and the result is that a solid phase is distributed everywhere in the design domain, the maximal feature in the void regions is smaller than the specified structural element.

The erosion and the dilation can be modeled using Heaviside projections with thresholds $\eta=0$ and $\eta=1$ and filter with finite support coinciding with the desired structural element. The eroded design will be denoted with $\bar{\rho}_{e}=\mathrm{H}(\widetilde{\bar{\rho}}, \eta=1)$ and the dilated with $\bar{\rho}_{d}=\mathrm{H}(\widetilde{\bar{\rho}}, \eta=0)$. It should be noted that the erosion and the dilation operations are applied on the filtered field $\widetilde{\bar{\rho}}$ obtained by filtering the physical design $\bar{\rho}$. The conditions for ensuring maximum length scale on the solid can be written as

$$
\int_{\Omega} \bar{\rho}_{e} \mathrm{~d} \mathbf{x}=0
$$

and the condition for ensuring maximum length scale on the void is given as

$$
\int_{\Omega}\left(1-\bar{\rho}_{d}\right) \mathrm{d} \mathbf{x}=0
$$


Taking into account the two additional constraints leads to the following modified minimum compliance optimization problem

$$
\begin{aligned}
& \min _{\rho}: J=\mathbf{f}^{\top} \mathbf{u} \\
& \text { s.t. }: \mathbf{K}(\boldsymbol{\rho}) \mathbf{u}=\mathbf{f} \\
& \overline{\boldsymbol{\rho}}_{e}^{\top} \mathbf{v} \leq \varepsilon_{s}|\mathbf{v}| \\
&\left(1-\overline{\boldsymbol{\rho}}_{d}\right)^{\top} \mathbf{v} \leq \varepsilon_{v}|\mathbf{v}| \\
& \overline{\boldsymbol{\rho}}^{\top} \cdot \mathbf{v} \leq V^{*} \\
& \mathbf{0} \leq \boldsymbol{\rho} \leq \mathbf{1}
\end{aligned}
$$

where $\varepsilon_{s}$ and $\varepsilon_{v}$ are small positive parameters used for relaxing slightly the problem. The two constraints can be combined with any of the approaches for imposing minimum length scale or with the robust formulation. An example for topology optimized design with maximum length scale on the solid region and minimum length scale on both phases is shown in Figure 5

Fig. 5 MBB beam designs with maximum length scale imposed on the solid regions and minimum length scale imposed on both phases.

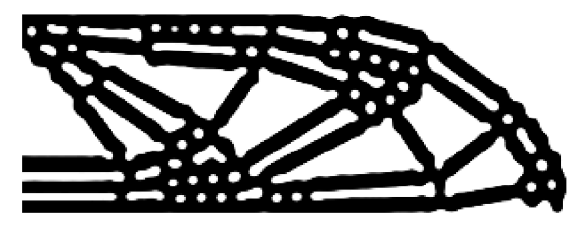

For level set approaches maximum length scale control is proposed in [106] and [6. Another attempt based on skeleton extraction is shown in 68. The idea is further developed in the following two papers 161 and 153. The main weakness in the latter formulations is that all of them disregard the gradients of the skeleton in the sensitivity analysis.

\section{Computational cost}

One of the main issues in density based topology optimization is the large number of optimization iterations. Each of them require a system solve, which for large scale problems can be time consuming. This is unacceptable and a large number of solutions have been proposed in the literature. As the state solvers can take more than $99 \%$ of the total computational time [2, the main focus has been in speeding up their solution time. The computational time can be decreased by improving the state solver's complexity, by parallelization of the algorithms, by reformulation of the optimization problems or decreasing the number of optimization steps, or by combination of any of the previous approaches.

Traditionally the solution of the system of equations obtained by discretization of the state problem is obtained by direct solvers due to their robustness. Their 
main limitations are the large amount of memory required during the factorization phase, the large computational complexity, and the lack of parallel scalability. Iterative solvers provide a solution to the above limitations, however they lack the robustness of the direct solvers. They rely on preconditioners in order to ensure convergence. Therefore, the weight in iterative solver development is on the construction of effective preconditioners. For elliptic problems the most effective preconditioners, as well as solvers, are based on multigrid techniques 142. Multigrid solvers are very effective for problems with smooth solutions and material properties and one of the main arguments against their use in topology optimization has been the high contrast in the material properties of the optimized designs. It should be pointed out that standard geometric multigrid methods are convergent 142 even though the convergence rate is affected by the high contrast in the material parameters. Their effectiveness is demonstrated in [1] and 1]. Other alternatives which have been demonstrated to be very effective not only for elliptic but also for wave propagation problems are domain decomposition techniques [4]. Recently contrast independent preconditioners utilizing ideas both from domain decomposition and multigrid have been presented in 89. The preconditioners have been applied in the design of robust manufacturable design with full resolution of the microstructural details 4, 3. The solvers have provided an optimized solution overnight on a single CPU for several million degrees of freedom. This is achieved by reusing the preconditioners between the different realizations in the robust formulation 13 and during the optimization between the different optimization steps. Another example for effective recycling of preconditioners is shown recently in [10].

The possibility of re-utilizing preconditioners between the different optimization steps 12 is based on the fact that the design changes slowly between updates. Furthermore, the solution tolerance can often be reduced for intermediate optimization steps [11, which is a still widely unexplored area. From a SAND (Simultaneous Analysis and Design [15]) formulation point of view the reduced tolerance for the intermediate solves can be seen as an inexact solves to the saddle point system arising from the formulation. It has been demonstrated in 119 that the SAND formulation leads to a very robust optimization process, however, an effective preconditioners for the saddle point system is necessary to be developed. Other computationally effective optimization formulations have been demonstrated in 47. and 48. They are applicable only for specific problems and are difficult to generalize. However, the examples demonstrate clearly the potential gains from reformulating the optimization problems.

An optimization based on the three field representation requires specification of the Heaviside projection relaxation parameter $\beta$. The optimization usually starts with small $\beta$ close to zero, and after convergence for the selected value, the relaxation parameter $\beta$ is increased. Such a continuation strategy may result in a divergence of the optimization process for large increments. For small increments the number of optimization iterations might be unnecessarily increased. Selecting close to optimal continuation strategy is an open question. A formulation which avoids the continuation and utilizes fixed $\beta$ is proposed in 60 .

The widely utilized MMA optimizer 133 is a first order optimization method which in general leads to large number of optimization steps resulting in larger number of system solves. A number of alternatives have been compared in [119] and the conclusion is that general non-linear optimization solvers, like IPOPT 
[143, often outperform MMA and its globally convergent counterpart GCMMA 134. Hence, the best optimization solver for density based topology optimization problems is still to be developed and is subject to active research.

\section{Summary}

The article aggregates the available techniques for obtaining manufacturable designs from density based topology optimization processes. The recent developments remove the post processing steps, thus, shortening the link between the design phase and the manufacturing phase. Incorporating the manufacturing process in the optimization formulation in some cases provides physical meaning for the originally pure mathematical entities utilized in the parametrization of the designs. Such an extension ensures manufacturability of the obtained designs and defines the design in terms of control parameters supplied to the manufacturer rather than a pure geometrical description. Furthermore, it removes not only the post processing step applied on the designer side but also the pre-processing manufacturing step, e.g., optical proximity correction in photolithography and proximity effect correction in EBL. In addition to the manufacturability, incorporating the control parameters in the optimization provides better management of the production cost and time. The similarities between the parametrization in density based topology optimization and nano- and micro-scale fabrication processes have been discussed for several of them. Further extensions and modifications remain to be seen for others.

Ensuring manufacturability does not guarantee well performing designs due to inevitable uncertainties in manufacturing. Hence, the optimization process needs to account for these uncertainties in order to reduce the waste products. The theoretical progress in this direction has been significant with a verification example in material design which demonstrates the removal of the post processing phases between topology optimization and manufacturing. Other verification examples will further strengthen the theoretical bases and will provide new development directions. Wider acceptance in industrial settings will be ensured by solving the high computational cost issues. A step in this direction is demonstrated here for minimum compliance optimal designs.

Often, due to lack of information, knowledge and experience, production and exploitation uncertainties cannot be supplied to the designer. In such cases rough manufacturing constraints, like minimum or maximum length scale, entirely based on engineering intuition, can be applied in the optimization process. Most of the constraints can be formulated as a single additional constraint to the topology optimization problem, thus, providing relatively cheap solutions from a computational point of view. For compliance design the minimum length scale constraint provides some robustness for the design performance, however, for wave propagation and compliant mechanism optimization problems such behavior is not guaranteed. On the other hand restricting the design space, by enforcing minimum length scale on the design, speeds up the optimization process significantly and can be utilized to ensure existence of solutions. The broad range of other manufacturing constraints makes the development, implementation and coordination with already developed techniques difficult. Their unification and the expansion of the already relatively wide set of constraints will be a subject of future research. 
Acknowledgements The authors acknowledge the financial support received from the NextTop project, sponsored by the Villum foundation and the Hypercool project sponsored by the Innovation Fund Denmark.

\section{References}

1. Aage, N., Andreassen, E., Lazarov, B.: Topology optimization using petsc: An easy-to-use, fully parallel, open source topology optimization framework. Structural and Multidisciplinary Optimization 51(3), 565-572 (2015). DOI 10.1007/s00158-014-1157-0

2. Aage, N., Lazarov, B.: Parallel framework for topology optimization using the method of moving asymptotes. Structural and Multidisciplinary Optimization 47(4), 493-505 (2013). DOI 10.1007/s00158-012-0869-2

3. Alexandersen, J., Lazarov, B.: Tailoring macroscale response of mechanical and heat transfer systems by topology optimization of microstructural details. In: N.D. Lagaros, M. Papadrakakis (eds.) Engineering and Applied Sciences Optimization, Computational Methods in Applied Sciences, vol. 38, pp. 267-288. Springer International Publishing (2015). DOI 10.1007/978-3-319-18320-6_15

4. Alexandersen, J., Lazarov, B.S.: Topology optimisation of manufacturable microstructural details without length scale separation using a spectral coarse basis preconditioner. Computer Methods in Applied Mechanics and Engineering 290(0), 156 - 182 (2015). DOI http://dx.doi.org/10.1016/j.cma.2015.02.028

5. Allaire, G., Francfort, G.: A numerical algorithm for topology and shape optimization. In: M. Bendse, C. Soares (eds.) Topology Design of Structures, NATO ASI Series, vol. 227, pp. 239-248. Springer Netherlands (1993). DOI 10.1007/978-94-011-1804-0_16. URL http://dx.doi.org/10.1007/978-94-011-1804-0_16

6. Allaire, G., Jouve, F., Michailidis, G.: Thickness control in structural optimization via a level set method (2014). URL https://hal.archives-ouvertes.fr/hal-00985000

7. Allaire, G., Jouve, F., Toader, A.M.: A level-set method for shape optimization. Comptes Rendus Mathematique 334(12), 1125 - 1130 (2002). DOI http://dx.doi.org/10.1016/ S1631-073X(02)02412-3

8. Allaire, G., Jouve, F., Toader, A.M.: Structural optimization using sensitivity analysis and a level-set method. Journal of Computational Physics 194(1), 363 - 393 (2004). DOI http://dx.doi.org/10.1016/j.jcp.2003.09.032

9. Allaire, G., Kohn, R.: Topology optimization and optimal shape design using homogenization. In: M. Bendse, C. Soares (eds.) Topology Design of Structures, NATO ASI Series, vol. 227, pp. 207-218. Springer Netherlands (1993). DOI 10.1007/978-94-011-1804-0_14. URL http://dx .doi.org/10.1007/978-94-011-1804-0_14

10. Amir, O.: Revisiting approximate reanalysis in topology optimization: on the advantages of recycled preconditioning in a minimum weight procedure. Structural and Multidisciplinary Optimization 51(1), 41-57 (2015). DOI 10.1007/s00158-014-1098-7

11. Amir, O., Aage, N., Lazarov, B.: On multigrid-CG for efficient topology optimization. Structural and Multidisciplinary Optimization 49(5), 815-829 (2014). DOI 10.1007/ s00158-013-1015-5

12. Amir, O., Sigmund, O.: On reducing computational effort in topology optimization: how far can we go? Structural and Multidisciplinary Optimization 44(1), 25-29 (2011). DOI $10.1007 / \mathrm{s} 00158-010-0586-7$

13. Amir, O., Sigmund, O., Lazarov, B.S., Schevenels, M.: Efficient reanalysis techniques for robust topology optimization. Computer Methods in Applied Mechanics and Engineering 245246(0), 217 - 231 (2012). DOI 10.1016/j.cma.2012.07.008

14. Andreassen, E., Lazarov, B.S., Sigmund, O.: Design of manufacturable 3d extremal elastic microstructure. Mechanics of Materials 69(1), 1 - 10 (2014). DOI http://dx.doi.org/10. 1016/j.mechmat.2013.09.018

15. Arora, J., Wang, Q.: Review of formulations for structural and mechanical system optimization. Structural and Multidisciplinary Optimization 30(4), 251-272 (2005). DOI 10.1007/s00158-004-0509-6

16. Aubert, G., Kornprobst, P.: Mathematical Problems in Image Processing: Partial Differential Equations and the Calculus of Variations, 2 edn. Springer (2006)

17. Bendsøe, M.P.: Optimal shape design as a material distribution problem. Structural optimization 1(4), 193-202 (1989). DOI 10.1007/BF01650949 
18. Bendsøe, M.P., Kikuchi, N.: Generating optimal topologies in structural design using a homogenization method. Computer Methods in Applied Mechanics and Engineering 71(2), 197 - 224 (1988). DOI 10.1016/0045-7825(88)90086-2. URL http://www.sciencedirect.com/science/article/pii/0045782588900862

19. Bendsøe, M.P., Sigmund, O.: Material interpolation schemes in topology optimization. Archive of Applied Mechanics 69, 635-654 (1999)

20. Bendsøe, M.P., Sigmund, O.: Topology Optimization - Theory, Methods and Applications. Springer Verlag, Berlin Heidelberg (2003)

21. Beyer, H.G., Sendhoff, B.: Robust optimization - a comprehensive survey. Computer Methods in Applied Mechanics and Engineering 196(33-34), 3190 - 3218 (2007). DOI DOI:10.1016/j.cma.2007.03.003

22. Borrvall, T., Petersson, J.: Topology optimization using regularized intermediate density control. Computer Methods In Applied Mechanics And Engineering 190(37-38), 49114928 (2001)

23. Bourdin, B.: Filters in topology optimization. International Journal for Numerical Methods in Engineering 50, 2143 - 2158 (2001)

24. Bourdin, B., Chambolle, A.: Design-dependent loads in topology optimization. ESAIM: Control, Optimisation and Calculus of Variations 9, 19-48 (2003). DOI 10.1051/cocv: 2002070

25. Brackett, D., Ashcroft, I., Hague, R.: Topology optimization for additive manufacturing. In: Proc of the 22nd Annual International Solid Freeform Fabrication Symposium (2011)

26. Brunner, T.A., Ferguson, R.A.: Approximate models for resist processing effects. In: Proc. SPIE, vol. 2726, pp. 198-207 (1996). DOI 10.1117/12.240906

27. Bruns, T.E., Tortorelli, D.A.: Topology optimization of non-linear elastic structures and compliant mechanisms. Computer Methods in Applied Mechanics and Engineering 190, 3443-3459 (2001)

28. Bückmann, T., Stenger, N., Kadic, M., Kaschke, J., Frölich, A., Kennerknecht, T., Eberl, C., Thiel, M., Wegener, M.: Tailored 3d mechanical metamaterials made by dip-in directlaser-writing optical lithography. Advanced Materials 24(20), 2710-2714 (2012). DOI 10.1002/adma.201200584

29. Chang, T.H.P.: Proximity effect in electronbeam lithography. Journal of Vacuum Science \& Technology 12(6), 1271-1275 (1975). DOI http://dx.doi.org/10.1116/1.568515. URL http://scitation.aip.org/content/avs/journal/jvst/12/6/10.1116/1.568515

30. Chen, S., Chen, W.: A new level-set based approach to shape and topology optimization under geometric uncertainty. Structural and Multidisciplinary Optimization 44(1), 1-18 (2011). DOI 10.1007/s00158-011-0660-9

31. Chen, S., Chen, W., Lee, S.: Level set based robust shape and topology optimization under random field uncertainties. Structural and Multidisciplinary Optimization 41, 507-524 (2010). DOI 10.1007/s00158-009-0449-2

32. Christiansen, R.E., Lazarov, B.S., Jensen, J.S., Sigmund, O.: Creating geometrically robust designs for highly sensitive problems using topology optimization. Structural and Multidisciplinary Optimization pp. 1-18 (2015). DOI 10.1007/s00158-015-1265-5

33. Clausen, A., Aage, N., Sigmund, O.: Topology optimization with flexible void area. Structural and Multidisciplinary Optimization 50(6), 927-943 (2014). DOI 10.1007/ s00158-014-1109-8

34. Clausen, A., Aage, N., Sigmund, O.: Topology optimization of coated structures and material interface problems. Computer Methods in Applied Mechanics and Engineering 290(0), 524 - 541 (2015). DOI http://dx.doi.org/10.1016/j.cma.2015.02.011

35. Coelho, P., Fernandes, P., Rodrigues, H., Cardoso, J., Guedes, J.: Numerical modeling of bone tissue adaptationa hierarchical approach for bone apparent density and trabecular structure. Journal of Biomechanics 42(7), 830 - 837 (2009). DOI 10.1016/j.jbiomech. 2009.01.020

36. Cui, Z.: Nanofabrication: Principles, Capabilities and Limits. Springer US (2008). DOI 10.1007/978-0-387-75577-9_2. URL http://dx.doi.org/10.1007/978-0-387-75577-9_2

37. Deaton, J.D., Grandhi, R.V.: A survey of structural and multidisciplinary continuum topology optimization: post 2000. Structural and Multidisciplinary Optimization 49(1), 1-38 (2014). DOI 10.1007/s00158-013-0956-z

38. Diaz, A., Sigmund, O.: Checkerboard patterns in layout optimization. Structural and Multidisciplinary Optimization 10, 40-45 (1995)

39. van Dijk, N., Maute, K., Langelaar, M., van Keulen, F.: Level-set methods for structural topology optimization: a review. Structural and Multidisciplinary Optimization 48(3), 437-472 (2013). DOI 10.1007/s00158-013-0912-y 
40. Dill, F., Neureuther, A.R., Tuttle, J., Walker, E.: Modeling projection printing of positive photoresists. Electron Devices, IEEE Transactions on 22(7), 456-464 (1975). DOI 10.1109/T-ED.1975.18161

41. Donoso, A., Sigmund, O.: Topology optimization of piezo modal transducers with nullpolarity phases. Structural and Multidisciplinary Optimization (2015). In print

42. Doyle, K.: Bioprinting: From patches to parts. Genetic Engineering \& Biotechnology News 34, 34-35 (2014). DOI 10.1089/gen.34.10.02

43. Dunning, P.D., Kim, H.A.: Robust topology optimization: Minimization of expected and variance of compliance. AIAA JOURNAL 51(11), 2656-2664 (2013). DOI 10.2514/1. J052183

44. Elesin, Y., Lazarov, B., Jensen, J., Sigmund, O.: Design of robust and efficient photonic switches using topology optimization. Photonics and Nanostructures - Fundamentals and Applications 10(1), 153 - 165 (2012). DOI 10.1016/j.photonics.2011.10.003

45. Elesin, Y., Lazarov, B., Jensen, J., Sigmund, O.: Time domain topology optimization of 3d nanophotonic devices. Photonics and Nanostructures - Fundamentals and Applications 12(1), 23 - 33 (2014). DOI http://dx.doi.org/10.1016/j.photonics.2013.07.008

46. Elishakoff, I., Ohsaki, M.: Optimization and Anti-optimization of Structures Under Uncertainty. Imperial College Press (2010)

47. Evgrafov, A.: State space newton's method for topology optimization. Computer Methods in Applied Mechanics and Engineering 278(0), 272 - 290 (2014). DOI http://dx.doi.org/ 10.1016/j.cma.2014.06.005

48. Evgrafov, A.: On chebyshevs method for topology optimization of stokes flows. Structural and Multidisciplinary Optimization pp. 1-11 (2015). DOI 10.1007/s00158-014-1176-x

49. Evgrafov, A., Rupp, C., Maute, K., Dunn, M.: Large-scale parallel topology optimization using a dual-primal substructuring solver. Structural and Multidisciplinary Optimization 36(4), 329-345 (2008). DOI 10.1007/s00158-007-0190-7

50. Frangopol, D.M., Maute, K.: Life-cycle reliability-based optimization of civil and aerospace structures. Computers \& Structures 81(7), 397 - 410 (2003). DOI http: //dx.doi.org/10.1016/S0045-7949(03)00020-8

51. Gan, Z., Cao, Y., Evans, R.A., Gu, M.: Three-dimensional deep sub-diffraction optical beam lithography with 9nm feature size. Nature Communications 4 (2013). DOI doi: $10.1038 /$ ncomms3061

52. Gaynor, A.T., Meisel, N.A., Williams, C.B., Guest, J.K.: Multiple-material topology optimization of compliant mechanisms created via polyjet three-dimensional printing. Journal of Manufacturing Science and Engineering 136, 061,015 (2014). DOI 10.1115/1. 4028439

53. Gaynor, A.T., Meisel, N.A., Williams, C.B., Guest, J.K.: Topology optimization for additive manufacturing: Considering maximum overhang constraint. In: AIAA Aviation, pp. . American Institute of Aeronautics and Astronautics (2014). DOI 10.2514/6.2014-2036. URL http://dx.doi.org/10.2514/6.2014-2036

54. Gersborg, A.R., Andreasen, C.S.: An explicit parameterization for casting constraints in gradient driven topology optimization. Structural and Multidisciplinary Optimization 44(6), 875-881 (2011). DOI 10.1007/s00158-011-0632-0

55. Ghanem, R., Spanos, P.: Stochastic Finite Elements - A Spectral Approach. Dover Publications Inc (2003)

56. Gibson, I., Rosen, D.W., Stucker, B.: Additive Manufacturing Technologies. Springer (2010)

57. Greengard, L., Rokhlin, V.: A fast algorithm for particle simulations. Journal of Computational Physics 73(2), 325 - 348 (1987). DOI http://dx.doi.org/10.1016/0021-9991(87) 90140-9

58. Groover, M.P.: Fundamentals of Modern Manufacturing: Materials, Processes, and Systems. John Wiley \& Sons, Inc. (2010)

59. Guest, J.: Imposing maximum length scale in topology optimization. Structural and Multidisciplinary Optimization 37, 463-473 (2009). URL http://dx.doi.org/10.1007/s00158-008-0250-7 10.1007/s00158-008-0250-7

60. Guest, J., Asadpoure, A., Ha, S.H.: Eliminating beta-continuation from heaviside projection and density filter algorithms. Structural and Multidisciplinary Optimization 44(4), 443-453 (2011). DOI 10.1007/s00158-011-0676-1

61. Guest, J., Prevost, J., Belytschko, T.: Achieving minimum length scale in topology optimization using nodal design variables and projection functions. International Journal for Numerical Methods in Engineering 61(2), 238-254 (2004) 
62. Guest, J.K.: Topology optimization with multiple phase projection. Computer Methods in Applied Mechanics and Engineering 199(1-4), 123 - 135 (2009)

63. Guest, J.K.: Optimizing the layout of discrete objects in structures and materials: A projection-based topology optimization approach. Computer Methods in Applied Mechanics and Engineering 283(0), 330 - 351 (2015). DOI http://dx.doi.org/10.1016/j. cma.2014.09.006

64. Guest, J.K., Ha, S.H.: Design of Multifunctional Material Structures Using Topology Optimization with Feature Control, pp. 129-134. John Wiley \& Sons, Inc. (2011). DOI 10.1002/9781118147726.ch17. URL http://dx.doi.org/10.1002/9781118147726.ch17

65. Guest, J.K., Prvost, J.H.: Optimizing multifunctional materials: Design of microstructures for maximized stiffness and fluid permeability. International Journal of Solids and Structures 43(2223), 7028 - 7047 (2006). DOI http://dx.doi.org/10.1016/j.ijsolstr.2006. 03.001

66. Guest, J.K., Smith Genut, L.C.: Reducing dimensionality in topology optimization using adaptive design variable fields. International Journal for Numerical Methods in Engineering 81(8), 1019-1045 (2010). DOI 10.1002/nme.2724

67. Guest, J.K., Zhu, M.: Casting and milling restrictions in topology optimization via projection-based algorithms. In: ASME 2012 International Design Engineering Technical Conferences and Computers and Information in Engineering Conference Volume 3: 38th Design Automation Conference, Parts A and B Chicago, Illinois, USA, August 1215 (2012)

68. Guo, X., Zhang, W., Zhong, W.: Explicit feature control in structural topology optimization via level set method. Computer Methods in Applied Mechanics and Engineering 272(0), 354 - 378 (2014). DOI 10.1016/j.cma.2014.01.010

69. Ha, S.H., Guest, J.: Optimizing inclusion shapes and patterns in periodic materials using discrete object projection. Structural and Multidisciplinary Optimization 50(1), 65-80 (2014). DOI 10.1007/s00158-013-1026-2. URL http://dx.doi.org/10.1007/s00158-013-1026-2

70. Haber, R., Jog, C., Bendsoe, M.: A new approach to variable-topology shape design using a constraint on perimeter. Structural Optimization 11(1), 1-12 (1996)

71. Hannon, A.F., Ding, Y., Bai, W., Ross, C.A., Alexander-Katz, A.: Optimizing topographical templates for directed self-assembly of block copolymers via inverse design simulations. Nano Letters 14(1), 318-325 (2014). DOI 10.1021/nl404067s. URL http://pubs.acs.org/doi/abs/10.1021/nl404067s

72. Harzheim, L., Graf, G.: A review of optimization of cast parts using topology optimization. Structural and Multidisciplinary Optimization 31(5), 388-399 (2006). DOI 10.1007/s00158-005-0554-9. URL http://dx.doi.org/10.1007/s00158-005-0554-9

73. Jansen, M., Lazarov, B.S., Schevenels, M., Sigmund, O.: On the similarities between micro/nano lithography and topology optimization projection methods. Structural and Multidisciplinary Optimization 48(4), 717-730 (2013). DOI 10.1007/s00158-013-0941-6

74. Jansen, M., Lombaert, G., Diehl, M., Lazarov, B.S., Sigmund, O., Schevenels, M.: Robust topology optimization accounting for misplacement of material. Structural and Multidisciplinary Optimization 47, 317-333 (2013). DOI 10.1007/s00158-012-0835-z

75. Jansen, M., Lombaert, G., Schevenels, M.: Robust topology optimization of structures with imperfect geometry based on geometric nonlinear analysis. Computer Methods in Applied Mechanics and Engineering 285(0), 452 - 467 (2015). DOI 10.1016/j.cma.2014. 11.028

76. Jansen, M., Lombaert, G., Schevenels, M., Sigmund, O.: Topology optimization of failsafe structures using a simplified local damage model. Structural and Multidisciplinary Optimization 49(4), 657-666 (2014). DOI 10.1007/s00158-013-1001-y

77. Jog, C.S., Haber, R.B.: Stability of finite element models for distributed-parameter optimization and topology design. Computer Methods In Applied Mechanics And Engineering 130(3-4), 203-226 (1996)

78. Kawamoto, A., Matsumori, T., Kondoh, T., Nomura, T., Yamasaki, S., Nishiwaki, S.: Topology optimization by using a time-dependent diffusion equation. In: Proc. of 9th World Congress on Structural and Multidisciplinary Optimization, Shizuoka, Japan (2011)

79. Kawamoto, A., Matsumori, T., Yamasaki, S., Nomura, T., Kondoh, T., Nishiwaki, S.: Heaviside projection based topology optimization by a pde-filtered scalar function. Structural and Multidisciplinary Optimization 44, 19-24 (2011). DOI 10.1007/ s00158-010-0562-2 
80. Kawata, S., Sun, H.B., Tanaka, T., Takada, K.: Finer features for functional microdevices. Nature 412, 697-698 (2001)

81. Kharmanda, G., Olhoff, N., Mohamed, A., Lemaire, M.: Reliability-based topology optimization. Structural and Multidisciplinary Optimization 26, 295-307 (2004)

82. Kim, T.S., Kim, J.E., Jeong, J.H., Kim, Y.Y.: Filtering technique to control member size in topology design optimization. KSME International Journal 18(2), 253-261 (2004). DOI 10.1007/BF03184735

83. Kogiso, N., Ahn, W., Nishiwaki, S., Izui, K., Yoshimura, M.: Robust Topology Optimization for Compliant Mechanisms Considering Uncertainty of Applied Loads. Journal of Advanced Mechanical Design Systems and Manifacturing 2(1), 96-107 (2008). DOI $10.1299 /$ jamdsm. 2.96

84. Kreisselmeier, G., Steinhauser, R.: Application of vector performance optimization to a robust control loop design for a fighter aircraft. International Journal of Control 37(2), 251-284 (1983). DOI 10.1080/00207179.1983.9753066

85. Landis, S. (ed.): Nano-Lithography. John Wiley \& Sons, Inc. (2013)

86. Lazarov, B., Matzen, R., Elesin, Y.: Topology optimization of pulse shaping filters using the hilbert transform envelope extraction. Structural and Multidisciplinary Optimization 44, 409-419 (2011). 10.1007/s00158-011-0642-y

87. Lazarov, B., Sigmund, O.: Sensitivity filters in topology optimisation as a solution to Helmholtz type differential equation. In: In Proc. of the 8th World Congress on Structural and Multidisciplinary Optimization (2009)

88. Lazarov, B., Wang, F.: Maximum length scale in density based topology optimization (2015). In review

89. Lazarov, B.S.: Topology optimization using multiscale finite element method for highcontrast media. In: I. Lirkov, S. Margenov, J. Waniewski (eds.) Large-Scale Scientific Computing, Lecture Notes in Computer Science, pp. 339-346. Springer Berlin Heidelberg (2014). DOI 10.1007/978-3-662-43880-0_38

90. Lazarov, B.S., Schevenels, M., Sigmund, O.: Robust design of large-displacement compliant mechanisms. Mechanical Sciences 2(2), 175-182 (2011). DOI 10.5194/ms-2-175-2011

91. Lazarov, B.S., Schevenels, M., Sigmund, O.: Topology optimization considering material and geometric uncertainties using stochastic collocation methods. Structural and Multidisciplinary Optimization 46, 597-612 (2012). 10.1007/s00158-012-0791-7

92. Lazarov, B.S., Schevenels, M., Sigmund, O.: Topology optimization with geometric uncertainties by perturbation techniques. International Journal for Numerical Methods in Engineering 90(11), 1321-1336 (2012). DOI 10.1002/nme.3361

93. Lazarov, B.S., Sigmund, O.: Filters in topology optimization based on Helmholtz-type differential equations. International Journal for Numerical Methods in Engineering 86(6), 765-781 (2011). DOI 10.1002/nme.3072

94. Le, C., Norato, J., Bruns, T., Ha, C., Tortorelli, D.: Stress-based topology optimization for continua. Structural and Multidisciplinary Optimization 41(4), 605-620 (2010). DOI 10.1007/s00158-009-0440-y. URL http://dx.doi.org/10.1007/s00158-009-0440-y

95. Leary, M., Merli, L., Torti, F., Mazur, M., Brandt, M.: Optimal topology for additive manufacture: A method for enabling additive manufacture of support-free optimal structures. Materials \& Design 63(0), 678 - 690 (2014). DOI http://dx.doi.org/10.1016/j. matdes.2014.06.015

96. Liu, J., Ma, Y.S.: 3d level-set topology optimization: a machining feature-based approach. Structural and Multidisciplinary Optimization pp. 1-20 (2015). DOI 10.1007/ s00158-015-1263-7

97. Liu, S., Li, Q., Chen, W., Hu, R., Tong, L.: H-DGTP-a heaviside-function based directional growth topology parameterization for design optimization of stiffener layout and height of thin-walled structures. Structural and Multidisciplinary Optimization pp. 1-11 (2015). DOI 10.1007/s00158-015-1281-5

98. Lógó, J., Ghaemi, M., Rad, M.: Optimal topologies in case of probabilistic loading: The influence of load correlation. Mechanics Based Design of Structures and Machines 37(3), 327-348 (2009)

99. Mack, C.: Fundamental Principles of Optical Lithography: The Science of Microfabrication. John Wiley \& Sons, Ltd (2007)

100. Markowitz, H.: Portfolio selection. The Journal of Finance 7(1), 77-91 (1952). DOI 10.1111/j.1540-6261.1952.tb01525.x

101. Maruo, S., Nakamura, O., Kawata, S.: Three-dimensional microfabrication with twophoton-absorbed photopolymerization. Opt. Lett. 22(2), 132-134 (1997). DOI 10.1364/ OL.22.000132 
102. Maute, K.: Topology optimization under uncertainty. In: G. Rozvany, T. Lewiski (eds.) Topology Optimization in Structural and Continuum Mechanics, CISM International Centre for Mechanical Sciences, vol. 549, pp. 457-471. Springer Vienna (2014). DOI 10. 1007/978-3-7091-1643-2_20. URL http://dx.doi.org/10.1007/978-3-7091-1643-2_20

103. Maute, K., Frangopol, D.: Reliability-based design of mems mechanisms by topology optimization. Computers and Structures 81, 813-824 (2003)

104. Mei, Y., Wang, X., Cheng, G.: A feature-based topological optimization for structure design. Advances in Engineering Software 39(2), 71 - 87 (2008). DOI http://dx.doi.org/ 10.1016/j.advengsoft.2007.01.023

105. Men, H., Freund, R.M., Nguyen, N.C., Saa-Seoane, J., Peraire, J.: Fabricationadaptive optimization with an application to photonic crystal design. Operations Research 62(2), 418-434 (2014). DOI 10.1287/opre.2013.1252. URL http://pubsonline.informs.org/doi/abs/10.1287/opre.2013.1252

106. Michailidis, G.: Manufacturing Constraints and Multi-Phase Shape and Topology Optimization via a Level-Set Method. Ph.D. thesis, Ecole Polytechnique X (2014). URL https://pastel.archives-ouvertes.fr/pastel-00937306

107. Mlejnek, H.: Some aspects of the genesis of structures. Structural optimization 5(1-2), 64-69 (1992). DOI 10.1007/BF01744697

108. Mohammad, M., Muhammad, M., Dew, S.K., Stepanova, M.: Fundamentals of electron beam exposure and development. In: M. Stepanova, S. Dew (eds.) Nanofabrication, pp. 11-41. Springer Vienna (2012). DOI 10.1007/978-3-7091-0424-8_2. URL http://dx.doi.org/10.1007/978-3-7091-0424-8_2

109. Najman, L., Talbot, H.: Mathematical Morphology: From Theory to Applications. John Wiley \& Sons, Inc. (2013). DOI 10.1002/9781118600788

110. Nomura, T., Sato, K., Taguchi, K., Kashiwa, T., Nishiwaki, S.: Structural topology optimization for the design of broadband dielectric resonator antennas using the finite difference time domain technique. International Journal for Numerical Methods in Engineering 71(11), 1261-1296 (2007). DOI 10.1002/nme.1974

111. Norato, J., Bell, B., Tortorelli, D.: A geometry projection method for continuum-based topology optimization with discrete elements. Computer Methods in Applied Mechanics and Engineering (0), - (2015). DOI http://dx.doi.org/10.1016/j.cma.2015.05.005. URL http://www.sciencedirect.com/science/article/pii/S0045782515001711

112. Oropallo, W., Piegl, L.: Ten challenges in 3d printing. Engineering with Computers pp. 1-14 (2015). DOI 10.1007/s00366-015-0407-0

113. Poonawala, A., Milanfar, P.: Mask design for optical microlithography mdash;an inverse imaging problem. Image Processing, IEEE Transactions on 16(3), 774-788 (2007). DOI 10.1109/TIP.2006.891332

114. Poulsen, T.A.: A new scheme for imposing a minimum length scale in topology optimization. International Journal for Numerical Methods in Engineering 57, $741-760$ (2003)

115. Qian, X., Sigmund, O.: Topological design of electromechanical actuators with robustness toward over- and under-etching. Computer Methods in Applied Mechanics and Engineering 253, 237-251 (2013). DOI 10.1016/j.cma.2012.08.020

116. Rehman, S., Langelaar, M., van Keulen, F.: Robust optimization of $2 \times 2$ multimode interference couplers with fabrication uncertainties. Proc. SPIE 8627, 862,713-862,713-11 (2013). DOI 10.1117/12.2000647. URL http://dx.doi.org/10.1117/12.2000647

117. Rehman, S., Langelaar, M., van Keulen, F.: Efficient kriging-based robust optimization of unconstrained problems. Journal of Computational Science 5(6), 872 - 881 (2014). DOI http://dx.doi.org/10.1016/j.jocs.2014.04.005. URL http://www.sciencedirect.com/science/article/pii/S1877750314000477

118. Rodrigues, H., Guedes, J., Bendsoe, M.: Hierarchical optimization of material and structure. Structural and Multidisciplinary Optimization 24(1), 1-10 (2002). DOI $10.1007 / \mathrm{s} 00158-002-0209-\mathrm{z}$

119. Rojas-Labanda, S., Stolpe, M.: Benchmarking optimization solvers for structural topology optimization. Structural and Multidisciplinary Optimization pp. 1-21 (2015). DOI 10.1007/s00158-015-1250-z. URL http://dx.doi.org/10.1007/s00158-015-1250-z

120. Ruszczynski, A., Shapiro, A.: Optimization of convex risk functions. Mathematics of operations research 31(3), 433-452 (2006). DOI 10.1287/moor.1050.0186

121. Saxena, A.: Topology design with negative masks using gradient search. Structural and Multidisciplinary Optimization 44(5), 629-649 (2011). DOI 10.1007/s00158-011-0649-4 
122. Schevenels, M., Lazarov, B., Sigmund, O.: Robust topology optimization accounting for spatially varying manufacturing errors. Computer Methods in Applied Mechanics and Engineering 200(49-52), 3613 - 3627 (2011). DOI 10.1016/j.cma.2011.08.006

123. Sigmund, O.: On the design of compliant mechanisms using topology optimization. Mechanics of Structures and Machines 25, 493-524 (1997)

124. Sigmund, O.: Morphology-based black and white filters for topology optimization. Structural and Multidisciplinary Optimization 33, 401-424 (2007). DOI 10.1007/ s00158-006-0087-x

125. Sigmund, O.: Manufacturing tolerant topology optimization. Acta Mechanica Sinica 25, 227-239 (2009)

126. Sigmund, O., Maute, K.: Topology optimization approaches. Structural and Multidisciplinary Optimization 48(6), 1031-1055 (2013). DOI 10.1007/s00158-013-0978-6

127. Sokolowski, J., Zochowski, A.: On the topological derivative in shape optimization. SIAM Journal on Control and Optimization 37(4), 1251-1272 (1999). DOI 10.1137/ S0363012997323230

128. Solomon, C., Breckon, T.: Fundamentals of Digital Image Processing: A Practical Approach with Examples in Matlab. John Wiley \& Sons, Ltd (2010)

129. Sørensen, R., Lund, E.: Thickness filters for gradient based multi-material and thickness optimization of laminated composite structures. Structural and Multidisciplinary Optimization pp. 1-24 (2015). DOI 10.1007/s00158-015-1230-3. URL http://dx.doi.org/10.1007/s00158-015-1230-3

130. Stolpe, M., Svanberg, K.: An alternative interpolation scheme for minimum compliance topology optimization. Structural and Multidisciplinary Optimization 22(2), 116-124 (2001). DOI 10.1007/s001580100129. URL http://dx.doi.org/10.1007/s001580100129

131. Stolpe, M., Svanberg, K.: On the trajectories of penalization methods for topology optimization. Structural and Multidisciplinary Optimization 21(2), 128-139 (2001). DOI 10.1007/s001580050177. URL http://dx.doi.org/10.1007/s001580050177

132. Suzuki, K.: Electron beam lithography systems. In: Microlithography, pp. 329-360-. CRC Press (2007). DOI 10.1201/9781420051537.ch6. URL http://dx.doi.org/10.1201/9781420051537.ch6

133. Svanberg, K.: The method of moving asymptotes - a new method for structural optimization. International Journal for Numerical Methods in Engineering 24, 359-373 (1987)

134. Svanberg, K.: A class of globally convergent optimization methods based on conservative convex separable approximations. SIAM Journal on Optimization 12, 555-573 (2002)

135. Svanberg, K., Svärd, H.: Density filters for topology optimization based on the pythagorean means. Structural and Multidisciplinary Optimization 48(5), 859-875 (2013). DOI 10.1007/s00158-013-0938-1

136. Takezawa, A., Kobashi, M., Kitamura, M.: Porous composite with negative thermal expansion obtained by photopolymer additive manufacturing (2015). URL http://arxiv.org/abs/1504.07724v1

137. Tomlin, M., Meyer, J.: Topology optimization of an additive layer manufactured (alm) aerospace part. In: The 7th Altair CAE technology conference (2011)

138. Tootkaboni, M., Asadpoure, A., Guest, J.K.: Topology optimization of continuum structures under uncertainty a polynomial chaos approach. Computer Methods in Applied Mechanics and Engineering 201-204(0), 263 - 275 (2012). DOI 10.1016/j.cma.2011.09.009

139. Travitzky, N., Bonet, A., Dermeik, B., Fey, T., Filbert-Demut, I., Schlier, L., Schlordt, T., Greil, P.: Additive manufacturing of ceramic-based materials. Advanced Engineering Materials 16(6), 729-754 (2014). DOI 10.1002/adem.201400097

140. Trefethen, L.N.: Spectral Methods in Matlab. SIAM (2000)

141. Tumbleston, J.R., Shirvanyants, D., Ermoshkin, N., Janusziewicz, R., Johnson, A.R., Kelly, D., Chen, K., Pinschmidt, R., Rolland, J.P., Ermoshkin, A., Samulski, E.T., DeSimone, J.M.: Continuous liquid interface production of 3d objects. Science 347(6228), 1349-1352 (2015). DOI 10.1126/science.aaa2397

142. Vassilevski, P.S.: Multilevel Block Factorization Preconditioners: Matrix-based Analysis and Algorithms for Solving Finite Element Equations. Springer, New York (2008)

143. Wächter, A., Biegler, L.T.: On the implementation of an interior-point filter line-search algorithm for large-scale nonlinear programming. Mathematical Programming 106(1), 25-57 (2006). DOI 10.1007/s10107-004-0559-y

144. Wadbro, E., Udawalpola, R., Berggren, M.: Shape and topology optimization of an acoustic hornlens combination. Journal of Computational and Applied Mathematics 234(6), 1781 - 1787 (2010). DOI http://dx.doi.org/10.1016/j.cam.2009.08.028 
145. Wang, F., Jensen, J.S., Sigmund, O.: Robust topology optimization of photonic crystal waveguides with tailored dispersion properties. J. Opt. Soc. Am. B 28(3), 387-397 (2011). DOI 10.1364/JOSAB.28.000387

146. Wang, F., Jensen, J.S., Sigmund, O.: High-performance slow light photonic crystal waveguides with topology optimized or circular-hole based material layouts. Photonics and Nanostructures - Fundamentals and Applications 10(4), $378-388$ (2012). DOI http://dx.doi.org/10.1016/j.photonics.2012.04.004. TaCoNa-Photonics 2011

147. Wang, F., Lazarov, B., Sigmund, O.: On projection methods, convergence and robust formulations in topology optimization. Structural and Multidisciplinary Optimization 43(6), 767-784 (2011). DOI 10.1007/s00158-010-0602-y

148. Wang, F., Lazarov, B.S., Sigmund, O., Jensen, J.S.: Interpolation scheme for fictitious domain techniques and topology optimization of finite strain elastic problems. Computer Methods in Applied Mechanics and Engineering 276(0), 453 - 472 (2014). DOI http: //dx.doi.org/10.1016/j.cma.2014.03.021

149. Wang, F., Sigmund, O., Jensen, J.S.: Design of materials with prescribed nonlinear properties. Journal Of The Mechanics And Physics Of Solids 69(1), 156-174 (2014). DOI 10.1016/j.jmps.2014.05.003

150. Wang, M., Zhou, S., Ding, H.: Nonlinear diffusions in topology optimization. Structural and Multidisciplinary Optimization 28, 262-276 (2004)

151. Wang, M.Y., Wang, X., Guo, D.: A level set method for structural topology optimization. Computer Methods in Applied Mechanics and Engineering 192(12), 227 - 246 (2003). DOI http://dx.doi.org/10.1016/S0045-7825(02)00559-5

152. Wu, S., Serbin, J., Gu, M.: Two-photon polymerisation for three-dimensional microfabrication. Journal of Photochemistry and Photobiology A: Chemistry 181(1), 1 - 11 (2006). DOI 10.1016/j.jphotochem.2006.03.004

153. Xia, Q., Shi, T.: Constraints of distance from boundary to skeleton: For the control of length scale in level set based structural topology optimization. Computer Methods in Applied Mechanics and Engineering 295, 525 - 542 (2015). DOI http://dx.doi.org/10. 1016/j.cma.2015.07.015

154. Xia, Q., Shi, T., Wang, M., Liu, S.: A level set based method for the optimization of cast part. Structural and Multidisciplinary Optimization 41(5), 735-747 (2010). DOI 10.1007/s00158-009-0444-7

155. Xia, Q., Shi, T., Wang, M., Liu, S.: Simultaneous optimization of cast part and parting direction using level set method. Structural and Multidisciplinary Optimization 44(6), 751-759 (2011). DOI 10.1007/s00158-011-0690-3

156. Xie, Y., Steven, G.: A simple evolutionary procedure for structural optimization. Computers \& Structures 49(5), 885 - 896 (1993). DOI http://dx.doi.org/10.1016/0045-7949(93) 90035-C

157. Xiu, D.: Numerical Methods for Stochastic Computations: A Spectral Method Approach. Princeton University Press (2010)

158. Xu, S., Cai, Y., Cheng, G.: Volume preserving nonlinear density filter based on heaviside functions. Structural and Multidisciplinary Optimization 41, 1615-1488 (2010)

159. Zauderer, E.: Partial Differential Equations of Applied Mathematics, 2 edn. (1998)

160. Zhang, W., Xia, L., Zhu, J., Zhang, Q.: Some recent advances in the integrated layout design of multicomponent systems. Journal of Mechanical Design 133, 15 (2011)

161. Zhang, W., Zhong, W., Guo, X.: An explicit length scale control approach in simpbased topology optimization. Computer Methods in Applied Mechanics and Engineering 282(0), 71 - 86 (2014). DOI 10.1016/j.cma.2014.08.027

162. Zhao, J., Wang, C.: Robust topology optimization under loading uncertainty based on linear elastic theory and orthogonal diagonalization of symmetric matrices. Computer Methods in Applied Mechanics and Engineering 273, $204-218$ (2014). DOI http://dx. doi.org/10.1016/j.cma.2014.01.018

163. Zhao, L., Ha, S., Sharp, K.W., Geltmacher, A.B., Fonda, R.W., Kinsey, A.H., Zhang, Y., Ryan, S.M., Erdeniz, D., Dunand, D.C., Hemker, K.J., Guest, J.K., Weihs, T.P.: Permeability measurements and modeling of topology-optimized metallic 3-d woven lattices. Acta Materialia 81(0), 326 - 336 (2014). DOI http://dx.doi.org/10.1016/j.actamat.2014. 08.037

164. Zhou, M., Fleury, R., Shyy, Y.K., Thomas, H., Brennan, J.: Progress in topology optimization with manufacturing constraints. In: 9th AIAA/ISSMO Symposium on Multidisciplinary Analysis and Optimization (2002). DOI 10.2514/6.2002-5614 
165. Zhou, M., Lazarov, B.S., Sigmund, O.: Topology optimization for optical projection lithography with manufacturing uncertainties. Appl. Opt. 53(12), 2720-2729 (2014). DOI 10.1364/AO.53.002720

166. Zhou, M., Lazarov, B.S., Wang, F., Sigmund, O.: Minimum length scale in topology optimization by geometric constraints. Computer Methods in Applied Mechanics and Engineering (2015). In print

167. Zhou, M., Rozvany, G.: The COC algorithm, part ii: Topological, geometrical and generalized shape optimization. Computer Methods in Applied Mechanics and Engineering 89(13), 309 - 336 (1991). DOI http://dx.doi.org/10.1016/0045-7825(91)90046-9

168. Zhou, M., Wang, M.Y.: Engineering feature design for level set based structural optimization. Computer-Aided Design 45(12), 1524 - 1537 (2013). DOI http://dx.doi.org/ 10.1016/j.cad.2013.06.016

169. Zhu, J.H., Gu, X.J., Zhang, W.H., Beckers, P.: Structural design of aircraft skin stretchforming die using topology optimization. Journal of Computational and Applied Mathematics 246, 278 - 288 (2013). DOI http://dx.doi.org/10.1016/j.cam.2012.09.001

170. Zhu, J.H., Zhang, W.H., Xia, L.: Topology optimization in aircraft and aerospace structures design. Archives of Computational Methods in Engineering pp. 1-28 (2015). DOI 10.1007/s11831-015-9151-2. URL http://dx.doi.org/10.1007/s11831-015-9151-2

171. Zienkiewicz, O.C., Taylor, R.L., Zhu, J.: The Finite Element Method. Elsevier (2005) 